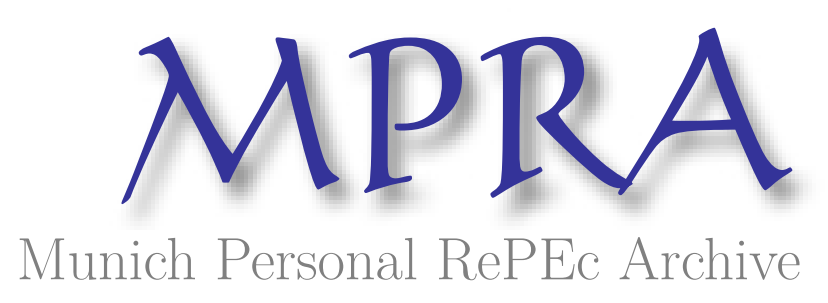

\title{
Mechanisms for Combinatorial Auctions with Budget Constraints
}

Le, Phuong

Stanford University

11 July 2015

Online at https://mpra.ub.uni-muenchen.de/66292/

MPRA Paper No. 66292, posted 28 Aug 2015 05:15 UTC 


\title{
Mechanisms for Combinatorial Auctions with Budget Constraints ${ }^{\text {th }}$
}

\author{
Phuong Le ${ }^{1}$
}

Stanford University

\begin{abstract}
This paper studies combinatorial auctions with budget-constrained bidders from a mechanism design perspective. I search for mechanisms that are incentive compatible, individually rational, symmetric, nonwasteful and non-bossy. First focusing on the greedy domain, in which any increase in a bidder's valuation always exceeds his budget, I derive the unique mechanism, called the Iterative Second Price Auction. For the general domain, however, no such mechanism exists.
\end{abstract}

Keywords: Combinatorial Auctions, Budget Constraints, Mechanisms

JEL Classification: D44, D82

\section{Introduction}

The progress of information technology brings ever increasing demand for telecommunications, by both end users and by industries. To meet this demand, telecommunications companies (telecoms) need to acquire more licenses for radio frequency spectrum. These licenses have been typically auctioned by the government to the telecoms. The Federal Communications Commission (FCC) spectrum auction in 1994 (Milgrom, 2000) is a prominent example. What makes spectrum auctions special is the combinatorial nature of the licenses: the value of a license depends on how it is combined with other licenses. For example, to some telecom, a license for a spectrum in California is worth $\$ 1$ million and a license for a spectrum in Nevada is also worth $\$ 1$ million, yet the combination of both licenses is worth $\$ 5$ million because the telecom can share infrastructure in the two states and reap economies of scale. Spectrum auctions are being implemented in many countries, yet many features of existing formats are not fully understood and many issues remain unresolved.

Spectrum auctions belong to the general class of combinatorial auctions. A particularly relevant issue in combinatorial auctions is that of budget constraints: bidders in the auction are constrained by their budgets. Continuing from the example above, the firm may be able to pay only $\$ 1$ million even if it gets both licenses. The presence of budget constraints for the bidders has been detected in many auctions (Bulow et al, 2009) and is therefore a very real concern. One immediate question is whether existing auction formats can accommodate budget constraints and if not, what modifications are needed.

\footnotetext{
This research was funded by the Leonard W. Ely and Shirley R. Ely Graduate Student Fund Fellowship from the Stanford Institute for Economic Policy Research.

Email address: phuong. le@alumni.stanford.edu (Phuong Le)

URL: https://sites.google.com/site/lp3ides (Phuong Le)

${ }^{1}$ I want to thank Fuhito Kojima, Ilya Segal, Alex Wolitzky and Gabriel Carroll for their helpful comments on a version of this paper that was in my dissertation at Stanford University, and am grateful to two anonymous referees for their insightful comments.
} 
The study of budget constraints in auction was first done in the single-unit setting by Che and Gale (1996) who show that all-pay auctions generate more revenue than first-price sealed-bid auctions. An optimal mechanism is found by Che and Gale (2000). When budgets are common knowledge, Laffront and Robert (1996) characterize the optimal auction as an all-pay auction with the proper reserve price. Much of later work focuses on the multi-unit setting where multiple copies of the same good are sold. Dobzinski et al (2008) show that when bidders are budget-constrained there is no incentive-compatible Pareto-optimal auction. They propose the adaptive clinching auction, a modification of the clinching auction in Ausubel (2004), and show that it satisfies Pareto-optimality, individual rationality and incentive compatibility when budgets are known. They also show that the adaptive clinching auction is in fact the only such mechanism. Bhattacharya et al (2010) show that when there is one infinitely divisible good, under-reporting budget is sub-optimal and the adaptive clinching auction is incentive compatible when over-reporting budget is either not possible (for example, when funds must be shown up-front) or made suboptimal through randomization. Also considering a divisible good, Hafalir et al (2011) propose a generalization of the Vickrey auction called the Vickrey with Budgets and show that it yields good revenue and Pareto optimality properties. In Vickrey with Budgets, under-rerporting budget is shown to be weakly dominated; over-reporting budget may be beneficial but would lead to higher revenue for the auctioneer. Borgs et al (2005) prove that, in the case of two buyers and two units, there is no truthful auction that allocate goods to distinct bidders. The authors also design an asymptotically revenue-maximizing truthful mechanism which may allocate only some of the items.

In the general valuation environment, there is no mechanism that is Pareto Optimal and strategy-proof, even when budgets are public knowledge (Gagan Goel, 2012; Dobzinski et al, 2008; Fiat et al, 2011; R. Lavi, 2011). However, not much is known about what kinds of allocations are implementable in dominant strategy. In this paper I posit five criteria for a mechanism: incentive compatibility, individual rationality, symmetry, non-wastefulness and non-bossiness. The first two concepts are standard. Symmetry means that the identity of the bidders does not matter. Non-wastefulness basically prevents the mechanism from wasting goods, and can be thought of as a weak notion of efficiency. From a practical perspective, nonwastefulness is appealing since many allocation algorithms are non-wasteful (see Nisan and Ronen, 2007). Non-bossiness means that a losing bidder cannot change the allocation for other bidders with a report that keeps him losing. The concept of non-bossiness is important in many allocation problems (Satterthwaite and Sonnenschein, 1981). Many mechanisms for auctions, such as VCG or (minimum-revenue) core-selecting auctions (Day and Milgrom, 2008), satisfy non-bossiness. My notion of non-bossiness is weaker than the standard notion, which requires that a bidder (not necessarily a losing bidder) cannot change the allocation for other bidders with a report that keeps his own allocation unchanged.

I first restrict attention to the greedy domain, in which for each bidder the difference in any two bundles' valuations, whenever non-zero, is more than his budget. In other words, a bidder is always willing to pay his entire budget for an increase in valuation. In this restricted domain, loosely speaking, misreports of valuation that win a bidder more goods is always profitable, regardless of the resulting payment (as long as payment does not exceed his budget). Greediness thereby allows for a kind of separation between allocation and payment and thereby greatly simplify the analysis of incentives.

Remarkably, the five criteria completely pin down an unique mechanism, called the Iterative Second Price Auction (ISPA), for the greedy domain. In ISPA, allocation is determined by a simple procedure, loosely described as follows. The bidder with the highest budget goes first and picks the bundle he wants, then the bidder with the second highest budget goes next and picks the bundle he wants out of the remaining goods, and so on. The payment of ISPA is determined by the externality principle: a bidder $i$ pays the highest budget of the bidders who, in bidder $i$ 's absence, would win a bundle overlapping with bidder $i$ 's 
bundle. The similarity between ISPA and the ordinary second price auction and the manner in which budget in the former plays the role of valuation in the latter are striking.

The characterization for the greedy domain combines the five criteria and uses induction to infer the outcome at one preference profile from the outcome at another preference profile. Incentive compatibility requires that, if the outcome at a profile is known, then the outcomes at another profile must be such that bidders do not deviate from the first profile to the second and vice versa. This allows me to determine the outcome for different profiles involving the same number of bidders and goods. To determine the outcome for profiles involving more bidders and more goods, I proceed in two steps. In the first step, new bidders are added to the profile and non-bossiness is invoked to pin down the outcome. In the second step, new goods are added and non-wastefulness is applied to determine which bidders would win these goods. Finally, induction establishes that the outcome rule must apply for all profiles, regardless of the number of bidders or goods.

Moving beyond the greedy domain to the general domain where the valuation of a bundle to a bidder, though positive, might be less than the bidder's budget, I show a negative result: there is no mechanism satisfying the five criteria for the general domain. This impossibility implies that if the five criteria must be satisfied, the auctioneer should look for ways to eliminate budget constraints (through financing schemes, for example). If budget constraints are unavoidable, one or more criteria must be relaxed or randomized mechanisms must be used.

The paper is organized as follows. Section 2 describes the environment and the five criteria. Section 3 defines the greedy domain and proves the uniqueness of ISPA for the greedy domain. The impossibility result is shown in section 4 . Section 5 concludes and suggests directions for future research.

\section{Preliminaries}

\subsection{The environment}

There is a set of indivisible goods $G$ belonging to a seller $S$ to be allocated among the bidders in the set I. A generic good is denoted by $g$, a generic bidder by $i$. A bundle of goods is a subset of $G$. A feasible allocation $x=\left(x_{1}, x_{2}, \ldots, x_{I}\right)$ specifies that the bundle $x_{i}$ is allocated to bidder $i$ and satisfies $x_{i} \cap x_{j}=\emptyset$ for all $i \neq j$. Let $X$ be the set of feasible allocations, and $2^{G}$ be the power set of $G$, i.e., the set of all subsets of $G$.

A bidder $i$ 's valuation over the bundles are summarized by a function $u_{i}: 2^{G} \rightarrow \mathbb{R}^{+}$that maps each bundle to a non-negative real number. The valuation from the empty bundle is zero, i.e. $u_{i}(\emptyset)=0$ for all $i$. I assume each bidder only cares about his own bundle and write $u_{i}(x)$ to mean $u_{i}\left(x_{i}\right)$, so for allocations $x$ and $y, x_{i}=y_{i}$ implies $u_{i}(x)=u_{i}(y)$. A bidder $i$ also has a budget $b_{i} \in \mathbb{R}^{+}$. Given a budget $b_{i}$, bidder $i$ cannot make a payment greater than $b_{i}$, regardless of the bundle he gets. Bidder $i$ 's characteristics are summarized by $\left(u_{i}, b_{i}\right)$. A profile $(u, b)=\left(u_{i}, b_{i}\right)_{i \in I}$ summarizes the characteristics of all bidders. Let $U \times B$ denote the set of profiles.

Let $P=\left\{p: p=\left(p_{i}\right)_{i \in I} \in \mathbb{R}_{+}^{I}\right\}$ be the set of payment vectors. An outcome is a pair $(x, p) \in X \times P$ that specifies that bidder $i$ gets bundle $x_{i}$ at the price $p_{i}$. Given an outcome $(x, p)$, the payoff for bidder $i$ with characteristics $\left(u_{i}, b_{i}\right)$ is given by

$$
v_{i}(x, p)= \begin{cases}u_{i}(x)-p_{i} & \text { if } p_{i} \leq b_{i} \\ -\infty & \text { otherwise }\end{cases}
$$

When $i$ can afford payment $p_{i}$, then his payoff takes the usual quasilinear form. However, when he cannot afford payment, his payoff is negative infinity - his budget constraint cannot be violated. 
The seller's valuation is assumed to be identically zero. The payoff for the seller is the total payment $v_{S}(x, p)=\sum_{i \in I} p_{i}$.

Set notation will be used heavily from now on, so to avoid confusion I clarify my notation here. The number of elements in a set $\mathscr{S}$ is denoted by $|\mathscr{S}| ; y \subseteq x$ means that $y$ is a subset of $x ; y \subset x$ means that $y$ is a proper subset of $x$, i.e., $y \subseteq x$ and $y \neq x$. The union of two bundles $x$ and $y$ is denoted by $x+y$. The set of goods in $x$ but not in $y$ is denoted by $x-y$.

\subsection{Mechanisms}

By the revelation principle, I can restrict attention to direct mechanisms. A direct mechanism elicits valuations and budgets from the bidders and then maps each profile to an outcome using a function $\psi$ : $U \times B \rightarrow X \times P$. Note that I am considering deterministic mechanisms. In a direct mechanism, each bidder's strategy space is the space of his valuation and budget. It will be notationally convenient to split the outcome mapping $\psi$ into two parts: the mapping to allocations $\psi^{a}: U \times B \rightarrow X$ and the mapping to payments $\psi^{p}: U \times B \rightarrow P$. Bidder $i$ 's allocation and payment at profile $(u, b)$ shall be referred to as $\psi_{i}^{a}(u, b)$ and $\psi_{i}^{p}(u, b)$ respectively. I denote by $\psi_{S}^{a}(u, b)$ the set of goods not allocated to any bidder (and thus allocated to the seller).

I focus on mechanisms that satisfy five criteria: incentive compatibility, individual rationality, symmetry, non-wastefulness and non-bossiness. A mechanism is individually rational if each bidder's payoff is non-negative. This property ensures that bidders will weakly gain from participating in the mechanism. Mechanisms that are not individually rational may deter bidders from entry.

Definition 1. An mechanism $\psi(\cdot)$ is individually rational (IR) if for any profile $(u, b), v_{i}(\psi(u, b)) \geq 0$ for all $i$.

A mechanism is incentive compatible if it is in the interest of each bidder $i$ to report his valuations and budget truthfully, regardless of the reports of other bidders. An incentive compatible mechanism makes it easy for the bidders to play in the elicitation stage: just report truthfully. The presence of budget constraints, however, means that at certain profiles of valuation and budget where tie-breaking is unavoidable, truthful reporting is no longer optimal. For example, consider the simple case where there are two agents and one good. A natural candidate mechanism is the second price auction, where each bidder bids his maximum willingness and ability to pay for the good. This mechanism is incentive compatible for generic valuations and budgets, but is not when the budgets are equal since in that case an agent will want to over-state his budget by a bit and win. So a mechanism is required to be incentive compatible only at generic profiles of valuations and budget. Formally, a profile is generic if no two bidders have the same budgets.

Definition 2. A profile $(u, b)$ is generic if $b_{i} \neq b_{j}$ for all $i \neq j$.

In the following, $u_{-i}$ and $b_{-i}$ denote the valuations and budget profile, respectively, of bidders other than $i$.

Definition 3. An mechanism $\psi(\cdot)$ is incentive compatible (IC) if at any generic profile $(u, b)$, for any $i \in I$, for all $\hat{u}_{i}, \hat{b}_{i}, v_{i}\left(\psi\left(u_{-i}, u_{i}, b_{-i}, b_{i}\right)\right) \geq v_{i}\left(\psi\left(u_{-i}, \hat{u}_{i}, b_{-i}, \hat{b}_{i}\right)\right)$.

The notion of incentive compatibility used here is ex-post. Though relatively restrictive, this notion allows for tractability in the analysis. One can alternatively posit ex-ante or interim incentive compatibility as a criterion. However, doing so requires assuming a prior on the distribution of valuations and budgets. The multidimensionality of such a distribution also makes it difficult to analyze. 
While incentive compatibility imposes certain restrictions on the outcome for a bidder $i$ when he changes his report, it imposes no restrictions on his outcome when other bidders change their reports. For example, a bidder can change his report and thereby changing the allocation for other bidders while maintaining his own outcome. The bidder who can change the allocation of others without changing his own allocation is called bossy. A mechanism is non-bossy if no losing bidders are bossy.

Definition 4. A mechanism $\psi(\cdot)$ is non-bossy if for any bidder $i, \psi_{i}^{a}(u, b)=\psi_{i}^{a}\left(u_{-i}, \hat{u}_{i}, b_{-i}, \hat{b}_{i}\right)=\emptyset$ implies that $\psi_{j}^{a}(u, b)=\psi_{j}^{a}\left(u_{-i}, \hat{u}_{i}, b_{-i}, \hat{b}_{i}\right)$ for all $j$.

Symmetry requires that, roughly speaking, it is the valuation and budget of a bidder, not his identity, that matter to the mechanism. It means that if two bidders swapped their reports, then the mechanism should swap their allocations and payments. Of course, in cases where tie-breaking is inevitable, the bidders' identities can be used to break ties. Symmetry is therefore only required for generic profiles.

Definition 5. A mechanism $\psi(\cdot)$ is symmetric if given any generic profile $(u, b), \psi_{i}(u, b)=\psi_{j}(\hat{u}, \hat{b})$ whenever all of the following hold

- $\left(u_{i}, b_{i}\right)=\left(\hat{u}_{j}, \hat{b}_{j}\right)$,

- $\left(u_{j}, b_{j}\right)=\left(\hat{u}_{i}, \hat{b}_{i}\right)$, and

- $\left(u_{k}, b_{k}\right)=\left(\hat{u}_{k}, \hat{b}_{k}\right)$ for all $k \neq i, j$.

Definition 6. A mechanism $\psi(\cdot)$ is non-wasteful if at any profile $(u, b)$

- $g \subseteq \psi_{S}^{a}(u, b)$ only if $u_{i}\left(\psi_{i}^{a}(u, b)+g\right)=u_{i}\left(\psi_{i}^{a}(u, b)\right)$ for all $i$, and

- for any bidder $i, g_{i} \subseteq \psi_{i}^{a}(u, b)$ only if $u_{i}\left(\psi_{i}^{a}(u, b)\right)>u_{i}\left(\psi_{i}^{a}(u, b)-g_{i}\right)$.

Non-wastefulness is quite simple intuitively: a non-wasteful mechanism does not waste any good. The first part of the definition serves to prevent the mechanism designer from not allocating any good to any bidder. It says that the seller keeps a good only if the good yields zero marginal valuation to every bidder. The second part prevents the mechanism designer from allocating goods indiscrimately among bidders. It says that if a good is allocated to some bidder, it must be that the marginal valuation of that good to that bidder is not zero. Loosely speaking, non-wastefulness requires that goods are allocated to bidders to create value if possible. However, it is silent on the magnitude of the value so created. In particular, it does not require that the value created is maximized. In other words, non-wastefulness can be thought of as a very weak notion of efficiency.

\section{Characterization for the greedy domain}

\subsection{The greedy domain}

Informally, in the greedy domain a bidder has valuation and budget such that if any two bundles give him different valuations, then the magnitude of the difference is larger than his budget. In other words, a greedy bidder is always willing to pay his entire budget to win a bundle or switch to another bundle, as long as doing so increases his valuation.

Before I describe the greedy domain formally, it will be convenient to first define the maximally relevant bundle. Loosely speaking, a maximally relevant bundle from a bundle $g$ is the smallest bundle that maximizes valuation, where smallest is measured in terms of set inclusion. In other words, it is the bundle that achieves as much valuation as the entire bundle $g$ but does not contain goods yielding zero marginal valuation. 
Table 1: Illustration of maximally relevant bundle

\begin{tabular}{c|ccccccc|c} 
& \multicolumn{7}{|c|}{ Bundles } & Budget \\
& 1 & 2 & 3 & 12 & 13 & 23 & 123 & \\
\hline Valuation & 0 & 0 & 2 & 3 & 2 & 4 & 4 & 0.5
\end{tabular}

Definition 7. The maximally relevant bundle $V_{i}(\cdot)$ for a given valuation function $u_{i}$ is a correspondence $V_{i}: 2^{G} \rightarrow 2^{G}$ where for any $g \subseteq G, V_{i}(g)=\left\{x_{i} \subseteq g: u_{i}\left(x_{i}\right)=u_{i}(g)\right.$, and $u_{i}\left(x_{i}\right)>u_{i}\left(y_{i}\right)$ for all $\left.y_{i} \subset x_{i}\right\}$.

I refer to $V_{i}(g)$ as bidder $i$ 's maximally relevant bundle( $\left.s\right)$ from $g$. For example, $V_{i}(G)$ refers to the maximally relevant bundle from the entire set of goods $G$. Note that if $u_{i}(g)=0$ then $V_{i}(g)=\emptyset$. When there is no potential for confusion, I write $V_{i}$ to refer to the set of all non-empty maximally relevant bundles, i.e., $V_{i}=\left\{x_{i}: x_{i} \neq \emptyset, x_{i}=V_{i}(g)\right.$ for some $\left.g \subseteq G\right\}$. I call $V_{i}$ bidder $i$ 's value set. Note that non-wastefulness requires that bidder $i$ 's allocation $x_{i}$ must either be empty or in $i$ 's value set.

For an illustration, suppose that there are 3 goods labeled 1,2,3 and bidder $i$ has valuation as shown in table 1 . Then $V_{i}(\{1,2,3\})=\{\{2,3\}\}$ because the bundle $\{2,3\}$ yields as much valuation as the bundle $\{1,2,3\}$ and good 1 brings no marginal valuation to the bundle $\{2,3\}$. Similarly, $V_{i}(\{1,3\})=\{\{3\}\}$ and $V_{i}(\{1\})=\emptyset$. The bidder's value set is $V_{i}=\{\{3\},\{1,2\},\{2,3\}\}$.

In general, there is no guarantee that the maximally relevant bundle is unique. In table 1 , if $u_{i}(\{1,3\})$ were 4 instead of 2 , then $V_{i}(\{1,2,3\})=\{\{1,3\},\{2,3\}\}$, i.e., there are 2 maximally relevant bundles from the set of all 3 goods.

Definition 8. A bidder $i$ is greedy if

1. for any bundle $x_{i}$ and any bundle $y_{i}$, if $u_{i}\left(x_{i}\right)-u_{i}\left(y_{i}\right)>0$ then $u_{i}\left(x_{i}\right)-u_{i}\left(y_{i}\right)>b_{i}$.

2. for all $g \subseteq G, V_{i}(g)$ is unique.

An bidder is called greedy if two conditions are satisfied. First, the difference in valuation between any two bundles, if positive, is greater than his budget. Second, his maximally relevant bundle from any given set is always unique. The first condition is crucial and provides greediness the bite that I need for my analysis. The second condition serves to ensure that the best bundle for a bidder from any given set of good is unique. For now, the reader can think of this as an assumption on the valuation function. I will discuss a relaxation of this assumption in a later section.

For example, the bidder with valuation and budget in table 1 is greedy, because any increase in his valuation going from one bundle to another is more than his budget. If his budget were 1.5, however, he would not be a greedy bidder. Another simple example of greediness is that of additive valuation where the marginal valuation of each good exceeds budget. However, greediness allows for much more general valuations.

A profile is greedy if each bidder either is greedy or has zero valuation for all bundles. Non-wastefulness implies that, for any greedy profile $(u, b)$ a bidder $i$ is either losing or winning a bundle $x_{i} \in V_{i}$. It will be useful to note that $V_{i}(G)$ is the highest-valuation maximally relevant bundle - the best outcome for a bidder $i$ always involves winning $V_{i}(G)$.

The greedy domain can be thought of as somewhat opposite of the quasilinear domain. In the latter, the valuation of any bundle can never exceed budget - budget is essentially infinite. Therefore, for any bundle there is always a price at which the bidder is indifferent between getting the bundle and not. The gain from manipulation may be ambiguous for a bidder since he might win more goods, but pay higher prices. In the 
greedy domain, however, any increase in valuation always exceed the bidder's budget, so there is no price at which the bidder is indifferent between getting additional valuation and not. Therefore, manipulations that enable the bidder to achieve higher valuation are always profitable because each increase in valuation is worth more than the auctioneer can charge him. For this reason, it matters more which bundles are maximally relevant and less how much valuation the bundles yield. The analysis will henceforth hinge more on the manipulation of value sets and less on valuation functions. Greediness allows for a kind of separation between allocation and payment and thereby greatly simplify the analysis of incentives.

Let $\phi(\cdot)$ be a mechanism that is incentive compatible, individually rational, symmetric, non-wasteful and non-bossy for the greedy domain, assuming that such a mechanism exists. I now completely characterize $\phi(\cdot)$. For the rest of this section, genericity and greediness are assumed.

\subsection{Preliminary results}

Much of the earlier analysis focuses on the allocation part of the mechanism. The following lemmas state that, if at a profile an allocation is chosen, then at some other profile where a bidder's valuation or budget has changed, some certain allocation has to be chosen. Since in the greedy domain, a bidder's characteristic is captured by his budget and value set, much of the results will pertain to the change in allocation (if any) when either budget or value set is changed.

The notion of an available outcome will be very useful. An outcome is available to a bidder $i$ at a report of other bidders if there is some report by bidder $i$ for which the mechanism outputs the outcome.

Definition 9. An outcome $\left(x_{i}, p_{i}\right)$ is available to bidder $i$ at the report $\left(u_{-i}, b_{-i}\right)$ by other bidders if there is a report $\left(u_{i}, b_{i}\right)$ by bidder $i$ such that $\phi_{i}(u, b)=\left(x_{i}, p_{i}\right)$.

The notion of availability is useful in the following way: if an outcome is available to bidder $i$, then given any report $\left(u_{i}, b_{i}\right)$ by bidder $i$, the mechanism must make sure that bidder $i$ 's payoff, valued at the report $\left(u_{i}, b_{i}\right)$, is at least as much as the payoff from the available outcome, also valued at the report $\left(u_{i}, b_{i}\right)$. Lemma 1 captures this direct consequence of incentive compatibility.

Lemma 1. If $\left(x_{i}, p_{i}\right)$ is available to bidder $i$ at $\left(u_{-i}, b_{-i}\right)$, then for any report $\left(u_{i}, b_{i}\right)$ by bidder $i$, $v_{i}\left(\phi_{i}\left(u_{-i}, u_{i}, b_{-i}, b_{i}\right)\right) \geq v_{i}\left(x_{i}, p_{i}\right)$.

A corollary of lemma 1 is that if a bundle containing $V_{i}(G)$ is available and affordable to bidder $i$, then bidder $i$ must win $V_{i}(G)$.

Corollary 1. If $\left(x_{i}, p_{i}\right)$ is available to bidder $i$ at $\left(u_{-i}, b_{-i}\right)$, and the report $\left(u_{i}, b_{i}\right)$ is such that $V_{i}(G) \subseteq x_{i}$ and $v_{i}\left(x_{i}, p_{i}\right)>0$, then $\phi_{i}^{a}(u, b)=V_{i}(G)$.

Proof. By lemma $1, v_{i}\left(\phi_{i}(u, b)\right) \geq v_{i}\left(x_{i}, p_{i}\right)>0$, so $i$ must be winning at report $\left(u_{i}, b_{i}\right)$. Since $x_{i}$ contains $V_{i}(G)$ and is available to $i$, greediness implies that the only way to satisfy $v_{i}\left(\phi_{i}(u, b)\right) \geq v_{i}\left(x_{i}, p_{i}\right)$ is for $\phi_{i}^{a}(u, b)$ to contain $V_{i}(G)$. By non-wastefulness, $\phi_{i}^{a}(u, b)$ is exactly $V_{i}(G)$.

One implication of corollary 1 is that if a bidder is winning some bundle, then by declaring that bundle to be his only maximally relevant bundle, he must still win the bundle.

Corollary 2. If $\phi_{i}^{a}(u, b)=x_{i} \neq \emptyset$, then for any report $\left(\hat{u}_{i}, b_{i}\right)$ with $\hat{V}_{i}=\left\{x_{i}\right\}, \phi_{i}^{a}\left(u_{-i}, \hat{u}_{i}, b\right)=x_{i}$.

While corollary 2 describes what happens when a winning bidder "shrinks" his value set, lemma 2 describes what happens when a winning bidder "expands" his value set. If a bidder is winning some bundle, then by expanding his value set he must still be winning. 
Lemma 2. If $\phi_{i}^{a}(u, b) \neq \emptyset$, then for any report $\hat{u}_{i}$ with value set $\hat{V}_{i}$ such that $V_{i} \subseteq \hat{V}_{i}, \phi_{i}^{a}\left(u_{-i}, \hat{u}_{i}, b\right) \neq \emptyset$.

Note that lemma 2 does not say that the bidder still wins the same bundle; he can win a different bundle.

Proof. Suppose $\phi_{i}^{a}(u, b)=\left(x_{i}, p_{i}\right)$ where $x_{i} \in V_{i}$ and $p_{i} \leq b_{i}$. Because $\left(x_{i}, p_{i}\right)$ is available to $i$, at any report $\left(\hat{u}_{i}, b_{i}\right)$, it must be that $\hat{v}_{i}\left(\phi_{i}\left(u_{-i}, \hat{u}_{i}, b\right)\right) \geq \hat{v}_{i}\left(x_{i}, p_{i}\right) . x_{i} \in V_{i}$ and $V_{i} \subseteq \hat{V}_{i}$ imply that $x_{i} \in \hat{V}_{i}$. Therefore, $\hat{v}_{i}\left(x_{i}, p_{i}\right)>0$. So bidder $i$ must be winning.

Lemma 2 directly implies that if a bidder is losing, then by shrinking his value set he must not become winning.

Lemma 3. If $\phi_{i}^{a}(u, b)=\emptyset$, then for any $\hat{u}_{i}$ with value set $\hat{V}_{i} \subseteq V_{i}, \phi_{i}^{a}\left(u_{-i}, \hat{u}_{i}, b\right)=\emptyset$.

The next lemma states that if a bidder is losing at two reports corresponding to two value sets, then reporting a value set that "combines" the two original value sets will not make him a winning bidder.

Lemma 4. Suppose $V_{i}$ and $V_{i}^{\prime}$ are the value sets associated with valuations $u_{i}$ and $u_{i}^{\prime}$, and that $\phi_{i}^{a}\left(u_{-i}, u_{i}, b\right)=$ $\phi_{i}^{a}\left(u_{-i}, u_{i}^{\prime}, b\right)=\emptyset$. Consider a value set $\hat{V}_{i}$ such that for all $z \in \hat{V}_{i}$, either $z=x$ or $z=y$ or $z=x \cup y$ for some $x \in V_{i}$ and $y \in V_{i}^{\prime}$. For all $\hat{u}_{i}$ with value set $\hat{V}_{i}, \phi_{i}^{a}\left(u_{-i}, \hat{u}_{i}, b\right)=\emptyset$.

Proof. Suppose in negation that $\phi_{i}^{a}\left(u_{-i}, \hat{u}_{i}, b\right)=\left(x_{i}, p_{i}\right)$ where $x_{i} \in \hat{V}_{i}$ and $p_{i} \leq b_{i}$. So $\left(x_{i}, p_{i}\right)$ is available to $i$. By definition of $\hat{V}_{i}, x_{i}$ must contain either (1) an element from $V_{i}$ or (2) an element from $V_{i}^{\prime}$, which implies either (1) $v_{i}\left(x_{i}, p_{i}\right)>0$ or (2) $v_{i}^{\prime}\left(x_{i}, p_{i}\right)>0$. In case (1), availability of $\left(x_{i}, p_{i}\right)$ means that for the report $\left(u_{i}, b_{i}\right), v_{i}\left(\phi_{i}(u, b)\right) \geq v_{i}\left(x_{i}, p_{i}\right)>0$, so $i$ must be winning, contradicting the assumption. Similarly, in case (2), $i$ must be winning at the report $\left(u_{i}^{\prime}, b_{i}\right)$, also contradicting the assumption.

For bundles $x$ and $y$, define the marginal valuation of $x$ at $y$ for bidder $i$ as $M V_{i}(x, y)=u_{i}(x+y)-u_{i}(y)$. A bundle $x_{0}$ is said to be worthless to bidder $i$ if the marginal valuation of $x_{0}$ is always zero to bidder $i$, i.e., $M V_{i}\left(x_{0}, y\right)=0$ for all $y$. A bundle $x_{0}$ is called worthless if it is worthless to all bidders. The following lemma states that a bidder can win through manipulation, in addition to what he is already winning, a bundle that is worthless.

Lemma 5. If $\phi_{i}^{a}(u, b)=x_{i} \in V_{i}$ and the bundle $x_{0}$ is worthless, then for any $\hat{u}_{i}$ such that $\hat{V}_{i}(G)=\left\{x_{i}+x_{0}\right\}$, $\phi_{i}^{a}\left(u_{-i}, \hat{u}_{i}, b\right)=\left\{x_{i}+x_{0}\right\}$.

Though non-wastefulness ensures that bundle $x_{0}$ must be assigned to bidder $i$, it is not obvious that bidder $i$ wins $x_{i}$ as well. The intuition is that bidder $i$ can report a very high valuation for $x_{i}$ and a relatively low valuation for $x_{0}$ (while still being greedy). The high valuation and availability of $x_{i}$ guarantees that bidder $i$ wins at least $x_{i}$, and non-wastefulness guarantees that he wins $x_{0}$ as well.

Proof. Suppose that $\phi_{i}(u, b)=\left(x_{i}, p_{i}\right)$ where $x_{i} \in V_{i}$ and $p_{i} \leq b_{i}$. Construct the valuation $\tilde{u}_{i}$ with the value set $\tilde{V}_{i}=\left\{x_{i}, x_{0}, x_{i}+x_{0}\right\}$ and $\tilde{u}_{i}\left(x_{i}+x_{0}\right)=\tilde{u}_{i}\left(x_{i}\right)+\tilde{u}_{i}\left(x_{0}\right)$ and $\tilde{u}_{i}\left(x_{i}\right)-b_{i}>\tilde{u}_{i}\left(x_{0}\right)>b_{i}$. This construction means that bidder $i$ is greedy to get $x_{i}$ and $x_{0}$, and that bidder $i$ strictly prefers winning $x_{i}$ over $x_{0}$ even if he has to pay his entire budget to get $x_{i}$.

By availability of $\left(x_{i}, p_{i}\right)$ and construction of valuation $\tilde{u}_{i}, \tilde{v}_{i}\left(\phi_{i}\left(u_{-i}, \tilde{u}_{i}, b\right)\right) \geq \tilde{v}_{i}\left(x_{i}, p_{i}\right)=\tilde{u}_{i}\left(x_{i}\right)-p_{i}>$ $\tilde{u}_{i}\left(x_{i}\right)-b_{i}>\tilde{u}_{i}\left(x_{0}\right)$. So bidder $i$ must be winning, and he must win more than just $x_{0}$. He cannot win just $x_{i}$ because non-wastefulness requires that $x_{0}$ be given to bidder $i$. Given that his value set is $\tilde{V}_{i}=\left\{x_{i}, x_{o}, x_{i}+x_{0}\right\}$, he must win $x_{i}+x_{0}$. Suppose that he has to pay $p_{0} \leq b_{i}$. Then the outcome $\left(x_{i}+x_{0}, p_{0}\right)$ is available to bidder $i$. By corollary 1 , if bidder $i$ 's valuation is $\hat{u}_{i}$ such that $\hat{V}_{i}(G)=\left\{x_{i}+x_{0}\right\}$ then $i$ must win $\left\{x_{i}+x_{0}\right\}$. 
The results so far pertain mostly to value sets. The following lemmas relate to budgets. Lemma 6 says that a winning bidder can not become a losing bidder by increasing his budget. This is intuitive, since increasing one's budget increases purchasing power and should not affect payoff negatively. Consequently, a losing bidder cannot become winning by lowering his budget.

Lemma 6. If $\phi_{i}^{a}(u, b) \in V_{i}$, then for any $\hat{b}_{i}>b_{i}, \phi_{i}^{a}\left(u, b_{-i}, \hat{b}_{i}\right) \in V_{i}$. If $\phi_{i}^{a}(u, b)=\emptyset$ then for all $\hat{b}_{i}<b_{i}$, $\phi_{i}^{a}\left(u, b_{-i}, \hat{b}_{i}\right)=\emptyset$.

Proof. For the first part of the lemma, suppose that $\phi_{i}(u, b)=\left(x_{i}, p_{i}\right)$ where $x_{i} \in V_{i}$ and $p_{i} \leq b_{i}$. Since $\left(x_{i}, p_{i}\right)$ is available to $i$, at report $\left(u_{i}, \hat{b}_{i}\right)$ with $\hat{b}_{i}>b_{i}$, it holds that $v_{i}\left(\phi_{i}\left(u, b_{-i}, \hat{b}_{i}\right)\right) \geq v_{i}\left(x_{i}, p_{i}\right)>0$. So bidder $i$ must still be winning. The second part of the lemma is a direct consequence of the first.

The final lemma, lemma 7, says that if a lower budget bidder $j$ is winning a bundle that is valued by a higher budget bidder $i$, then bidder $i$ must also be winning. The intuition is simple: if bidder $i$ is not winning any bundle, he can, by virtue of his budget being higher, "pretend" to be bidder $j$ and win $j$ 's current bundle, thereby improving his payoff. This would violate IC. Note that the ability to pretend budget-wise is not symmetric: a lower budget bidder cannot always pretend to be a higher budget bidder if by doing so he will have to make payments exceeding his actual budget. One can think of this result as a kind of budget dominance.

Lemma 7. Suppose $b_{i}>b_{j}$. If $\phi_{j}^{a}(u, b) \in V_{i}$, then $\phi_{i}^{a}(u, b) \in V_{i}$.

ProOF. See appendix.

The proof is by contradiction. Starting from a proposed allocation that violates lemma 7, I show that certain allocations must hold at other profiles and eventually show that symmetry is violated. The formal proof is in the appendix, though the main idea may be illustrated using a "transition" table. In the tables, bidders are shown as rows, and goods are shown as columns. A box filled with the times sign $\times$ or the check mark $\checkmark$ in row $i$ and column $g$ means $g \in V_{i}$, and the absence of a box means $g \notin V_{i}$. For ease of illustration, the tables do not show all elements in the value set - it is implicit henceforth, and unless stated otherwise, that whenever table illustrations are used, unions bundles in the value set are also in the value set. For example, the bundle consisting a $x$ and a $\checkmark$ is in the value set. The reader can think of the valuation function as additive (but valuation function can take more general forms). A box filled with a check mark $\checkmark$ means $g$ is allocated to $i$. For example, the table under "Profile 1" in table 2 shows an allocation with 3 goods and 3 bidders where each bidder's value set contains all bundles. Allocation-wise, bidder 1 gets goods 1 and 2, while bidder 2 gets goods 3 , and bidder 2 is losing.

The proof can now be illustrated with table 2. Starting from a candidate allocation in profile 1 that violates lemma 7, I conclude what allocation must hold as certain changes in value set and budget are made sequentially, invoking the results established above. The violation of symmetry can be seen by comparing the allocations of profile 3 and profile 5 .

\subsection{Main results}

The problem of allocating $G$ to $I$ can be daunting if each bidder's allocation is dependent on other bidders'. Fortunately, non-wastefulness allows me to group bidders and goods into groups where bidders in a group only care about goods in the same group and these goods are valuable to these bidders only. Such a group is called an isolated group. Formally, 
Table 2: Transitions for 3 bidders and 3 goods

Profile 1

A candidate allocation that violates lemma 7

\begin{tabular}{|c|c|c|c|c|}
\hline & 1 & 2 & 3 & b \\
\hline 1 & $\checkmark$ & $\checkmark$ & $x$ & 15 \\
\hline 2 & $\times$ & $x$ & $x$ & 10 \\
\hline 3 & $\bar{x}$ & $x$ & $\sqrt{ }$ & 5 \\
\hline
\end{tabular}

Profile 3

Bidder 3 shrinks value set and remains winning, by corollary 2
Profile 2

Bidder 2 shrinks value set and remains losing, by lemma 3

\begin{tabular}{c|ccc|c|c} 
& 1 & 2 & 3 & $\mathrm{~b}$ \\
\hline 1 & $\checkmark$ & $\checkmark$ & $\times$ & 15 \\
2 & & & $\times$ & 10 \\
3 & $\times$ & $\times$ & $\checkmark$ & 5
\end{tabular}$\rightarrow$

Profile 4

Bidder 3 increases budget and remains winning, by lemma 6

\begin{tabular}{c|ccc|c} 
& 1 & 2 & 3 & $\mathrm{~b}$ \\
\hline 1 & $\checkmark$ & $\checkmark$ & $\times$ & 15 \\
2 & & & $\times$ & 10 \\
3 & & & $\checkmark$ & 10
\end{tabular}$\rightarrow$

Profile 5

Bidder 2 decreases budget and remains losing, by lemma 6

\begin{tabular}{c|cccc|c} 
& 1 & 2 & 3 & $\mathrm{~b}$ \\
\hline 1 & $\checkmark$ & $\checkmark$ & $\times$ & 15 \\
2 & & & $\times$ & 5 \\
3 & & & $\checkmark$ & 10
\end{tabular}


Definition 10. Given a profile $(u, b)$ with value sets $V_{I}=\left(V_{i}\right)_{i \in I},\left(I^{\prime}, G^{\prime}, V_{I^{\prime}}\right)$ is called an isolated group if $I^{\prime} \subseteq I$ is a set of bidders and $G^{\prime} \subseteq G$ is a set of goods such that

- For all goods $g \notin G^{\prime}$, for all bidders $i \in I^{\prime}$, for all bundles $y, M V_{i}(g, y)=0$.

- For all bidders $j \notin I^{\prime}$, for all goods $g \in G^{\prime}$, for all bundles $y, M V_{j}(g, y)=0$.

Basically, a group of bidders $I^{\prime}$ and goods $G^{\prime}$ is isolated if the bidders in $I^{\prime}$ value only the goods in $G^{\prime}$, and that the goods in $G^{\prime}$ are valued only by the bidders in $I^{\prime}$. As far as the bidders in $I^{\prime}$ are concerned, goods outside $G^{\prime}$ are irrelevant; as far as the bidders outside $I^{\prime}$ are concerned, goods inside $G^{\prime}$ are irrelevant. It is convenient to analyze isolated groups because, by non-wastefulness, goods within an isolated group can only be allocated to bidders in the same isolated group. In this sense, one can break down the problem of assigning $G$ to $I^{\prime}$ to the problem assigning goods to bidders in isolated groups. I will often simplify notation and write $\left(I^{\prime}, G^{\prime}, V\right)$ to mean $\left(I^{\prime}, G^{\prime}, V_{I^{\prime}}\right)$. Note that, by definition, the "grand coalition group" $(I, G, V)$ is an isolated group.

Perhaps the simplest structure for an isolated group is the rectangular structure, defined as follows.

Definition 11. An isolated group $\left(I^{\prime}, G^{\prime}, V\right)$ is called rectangular if $V_{i}=2^{G^{\prime}}-\emptyset$ for all $i \in I^{\prime}$.

In an isolated rectangular group $\left(I^{\prime}, G^{\prime}, V\right)$, for each bidder in $I^{\prime}$, his value set consists of all combinations of goods in $G^{\prime}$. The name "rectangular" comes from the shape of the value set when represented graphically, such as profile 1 in table 2 for a set of 3 bidders and 3 goods.

The first main result states that a rectangular isolated group, the bidder with the highest budget wins all the relevant goods.

Theorem 1. Let $\left(I^{\prime}, G^{\prime}, V\right)$ be a isolated rectangular group. If $b_{i}=\max _{j \in I^{\prime}} b_{j}$, then $\phi_{i}^{a}(u, b)=G^{\prime}$.

PROOF. See appendix for a formal proof. Here I sketch out the main steps. The proof uses induction on "triangular" profiles. A triangular profile is formally defined in the appendix, but for the time being the reader can get a sense of what "triangular" means by looking at the triangular profile in table 3 , as well as profile 1 in table 5. These profiles "look" like triangles, in the sense that the sets of individual goods in the respective value sets shrink by one good or "cascade" down the rows. Note that although profile 2 in table 5 does not look like a triangular, rearranging the good 2 and good 3 will make it triangular.

I first show that for any isolated group of triangular shape with 2 bidders and 2 goods, the highest budget bidder wins all goods in the group. I then proceed to show that triangular profiles can be "expanded" to obtain profiles of larger triangular shapes at which the highest budget bidder still wins all goods in the group. The same process of expansion can be used to fill out the triangle shape to arrive at a square shape, all the while maintaining the allocation where the highest budget bidder wins all the goods in the group. The rectangular shape can then be obtained by removing goods or bidders, as appropriate, from the group.

From now on I label bidders in the isolated group in decreasing order of their budget, so that $b_{1}>b_{2}>\ldots$ I begin with the base case of the triangle of size 2. Consider the isolated group in table 3 in which bidder 1 has the higher budget.

Step 1: Consider what happens if both bidders value only $g_{1}$. Because of non-wastefulness, $g_{1}$ must be allocated to either bidder 1 or bidder 2. By lemma 7, if bidder 2 wins then bidder 1 must win as well, but this is not possible. Therefore bidder 1 wins $g_{1}$.

Step 2: Back to the triangle. By step 1 and lemma 5, bidder 1 wins $g_{1}+g_{2}$. Therefore, $\phi_{1}^{a}(u, b)=V_{1}(G)$. This result is captured by table 4 . 
Table 3: The base case: "triangular" profile of size 2

\begin{tabular}{|c|c|c|}
\hline & $g_{2}$ & $g_{1}$ \\
\hline 1 & $x$ & $x$ \\
\hline 2 & & $\times$ \\
\hline
\end{tabular}

Table 4: Allocation of the base case

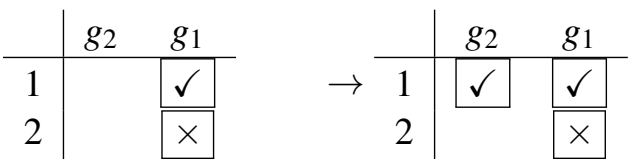

Now I illustrate how a triangle can be "expanded" to obtain a larger triangle. For concreteness and ease of illustration, I use the inductive hypothesis for a specific group size: at any isolated group with triangular shape of size 3, bidder 1 wins all goods in the group. The insight is that two such groups can be "superimposed" to arrive at the allocation of a group which is essentially a triangle to which a box has been added. Consider the profiles in table 5. Profile 1 and profile 2 are both triangular, and so by the inductive hypothesis bidder 1 wins all the goods. Lemma 4 implies then that when bidder 2 "combines" his value sets so that the group has the shape shown in profile 3, bidder 2 is still losing. By non-bossiness, bidder 1 still wins all goods in profile 3.

I can use the same superimposition technique to add a good to bidder 3's value set and preserve the allocation. Schematically, superimposition allows me to have the allocation shown in table 6 . Note that the newly added boxes have the "+" sign, + .

Next, consider a bidder 4 who does not value any good and must therefore be losing. Suppose I change bidder 4's valuation and budget such that he values good $g_{1}$ and his budget is the smallest, i.e., $b_{4}<b_{3}$. By lemma 7, if bidder 4 is winning $g_{1}$, then bidders $1,2,3$ must be winning since $g_{1}$ is in each bidder's value set and these bidders dominate bidder 4 budget-wise. However, there are then only 3 goods for 4 winning bidders, an impossibility. Therefore, bidder 4 is still losing. Non-bossiness then implies that bidder 1 still wins all the goods. The argument is summarized by table 7 .

Finally, to obtain a triangle, I modify bidder 1's value set so that his maximally relevant bundle $V_{1}(G)$ contains all 3 goods and an additional good $g_{4}$. By lemma 5, bidder 1 wins all 4 goods. See table 8 .

I have shown, albeit informally, that triangular profiles can be expanded yet preserve the particular allocation in which the highest budget bidder wins all goods in the isolated group. Since this allocation rule is true for triangles of size 2, induction can be use to establish that this allocation rule is true for triangles of all sizes.

It is easy to see that the superimposition technique can be use to fill out a triangular group to obtain a "square" group. Non-bossiness then guarantees that adding or removing losing bidders from a square group to arrive at a rectangular group does not change the allocation. Therefore, for any isolated rectangular group, the highest budget bidder wins all goods in the group.

The result that the bidder with the highest budget in an isolated group wins all the goods is not limited to rectangular groups. In fact, for any isolated group, the bidder with the highest budget wins his maximally relevant bundle from the group's set of goods.

Theorem 2. Let $\left(I^{\prime}, G^{\prime}, V\right)$ be an isolated group. If $b_{i}=\max _{j \in I^{\prime}} b_{j}$, then $\phi_{i}^{a}(u, b)=V_{i}\left(G^{\prime}\right)$. 
Table 5: Example of "superimposition"

Profile 1

By assumption

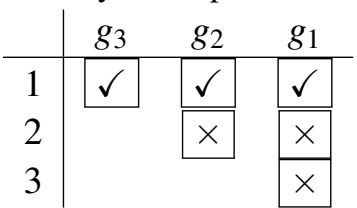

Profile 3

By lemma 4

\begin{tabular}{c|cc|c|} 
& $g_{3}$ & $g_{2}$ & $g_{1}$ \\
\hline 1 & $\checkmark$ & $\checkmark$ & $\checkmark$ \\
2 & $\times$ & $\times$ & $\times$ \\
\hline 3 & & & $\times$ \\
\hline & & &
\end{tabular}

Profile 2

By assumption

and

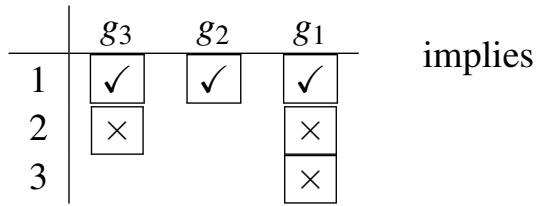

Table 6: Adding good to value set

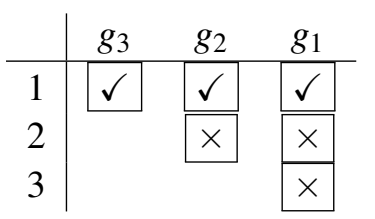

\begin{tabular}{c|ccc|} 
& $g_{3}$ & $g_{2}$ & $g_{1}$ \\
\hline 1 & $\checkmark$ & $\checkmark$ & $\checkmark$ \\
\hline 2 & $\checkmark$ & $\times$ & $\times$ \\
\hline 3 & & & $\times$ \\
\hline
\end{tabular}

\begin{tabular}{c|cc|c|} 
& $g_{3}$ & $g_{2}$ & $g_{1}$ \\
\hline 1 & $\checkmark$ & $\checkmark$ & $\checkmark$ \\
\hline 2 & $\times$ & $\times$ & $\times$ \\
\hline 3 & & + & $\times$ \\
\hline
\end{tabular}

Table 7: Adding a losing bidder

\begin{tabular}{|c|c|c|c|c|c|c|c|c|}
\hline & $g_{3}$ & $g_{2}$ & $g_{1}$ & & & $g_{3}$ & $g_{2}$ & $g_{1}$ \\
\hline 1 & $\checkmark$ & $\checkmark$ & $\checkmark$ & & 1 & $\checkmark$ & $\checkmark$ & $\checkmark$ \\
\hline 2 & $\times$ & $\bar{x}$ & $\times$ & & 2 & $\times$ & $x$ & $x$ \\
\hline 3 & & $\bar{x}$ & $\bar{x}$ & & 3 & & $x$ & $\bar{x}$ \\
\hline 4 & & & & & 4 & & & + \\
\hline
\end{tabular}

Table 8: Adding a good

\begin{tabular}{|c|c|c|c|c|c|c|c|c|c|c|}
\hline & $g_{4}$ & $g_{3}$ & $g_{2}$ & $g_{1}$ & & & $g_{4}$ & $g_{3}$ & $g_{2}$ & $g_{1}$ \\
\hline 1 & & $\bar{\checkmark}$ & $\checkmark$ & $\bar{\checkmark}$ & & 1 & $\checkmark$ & $\checkmark$ & $\checkmark$ & $\checkmark$ \\
\hline 2 & & $\bar{x}$ & $\times$ & $\times$ & & 2 & & $\times$ & $x$ & $\bar{x}$ \\
\hline 3 & & & $\times$ & $x$ & & 3 & & & $x$ & $x$ \\
\hline 4 & & & & $x$ & & 4 & & & & $x$ \\
\hline
\end{tabular}


PROOF. First consider a hypothetical group $\left(I^{\prime}, G^{\prime}, \hat{V}\right)$ where bidders' budgets are unchanged, i.e., $b_{i}^{\prime}=b_{i}$ for all $i$, but their value sets $\hat{V}_{I^{\prime}}$ are such that for all $i, \hat{V}_{i}=G^{\prime}$. Then $\left(I^{\prime}, G^{\prime}, \hat{V}\right)$ is an isolated rectangular group. By theorem 1, the bidder with the highest budget, labeled bidder 1, wins all the goods.

Now consider the change in value set for a bidder $j \neq 1$ from $\hat{V}_{j}$ to $V_{j}$. If bidder $j$ wins any good in $G^{\prime}$ with value set $V_{j}$, then when his value set is $\hat{V}_{j}$ he has a profitable deviation: report $V_{j}$ instead. Because this violates IC, bidder $j$ must still be losing when his reported value set is $V_{j}$. Non-bossiness implies that bidder 1 still wins all the goods after this change. By this reasoning, the allocation is preserved if I apply the change from $\hat{V}_{j}$ to $V_{j}$ for each bidder $j \neq 1$ sequentially, bidder by bidder. Hence, when value sets are $V_{j}$ for $j \neq 1$ and $\hat{V}_{1}$ for bidder 1 , bidder 1 wins all the goods. Suppose he pays $p_{1} \leq b_{1}$.

Now, change bidder $i$ 's value set from $\hat{V}_{1}$ to $V_{1}$. Because the outcome $\left(G^{\prime}, p_{1}\right)$ is available to bidder 1 , corollary 1 implies that bidder 1 wins $V_{1}\left(G^{\prime}\right)$.

Theorem 2 states that for any isolated group the bidder $i$ with the highest budget wins all goods in his value set. One can of course apply this result to the "grand coalition group" $(I, G, V)$. If the maximally relevant bundle from $G$ for bidder $i$ contains all goods, then the allocation part of the problem is done. However, if there are goods outside this bundle, then by non-wastefulness these goods must not be allocated to this bidder and must instead be allocated to other bidders or the seller. This problem is not as daunting as might at first seems, since it can be thought of as a smaller instance of the original allocation problem: allocate goods in the set $G^{\prime}=G-V_{i}(G)$ to bidders in the set $I^{\prime}=I-i$. Because $V_{i}(G)$ has been allocated to bidder $i$, the remaining bidders and the remaining goods are effectively isolated. An idea similar to theorem 2 can then be used for the remaining bidders and goods to argue that the bidder with the highest budget among the remaining bidders, say bidder $j$, wins $V_{j}\left(G^{\prime}\right)$, his maximally relevant bundle from $G^{\prime}$. This reasoning can be applied again and again until all goods are allocated. I summarize the result in the following theorem. From now on, I consider the seller as a bidder with zero valuation and zero budget.

Theorem 3. Let $(u, b)$ be given. The allocation $\phi^{a}(u, b)$ is given by the following procedure:

- Step 1:

$\diamond$ Label the set of remaining goods $G^{\prime}$ and the set of remaining bidders $I^{\prime}$.

$\diamond$ If $G^{\prime}=\emptyset$ then $\phi_{i}^{a}(u, b)=\emptyset$ for all $i \in I^{\prime}$ (all remaining bidders are losing) and terminate the procedure.

$\diamond$ If $I^{\prime}=\emptyset$ then stop the procedure.

$\diamond$ Let $i$ be the bidder with the highest budget, i.e., $b_{i}=\max _{j \in I^{\prime}} b_{j}$.

$\diamond \phi_{i}^{a}(u, b)=V_{i}\left(G^{\prime}\right)$.

$\diamond$ Remove $V_{i}\left(G^{\prime}\right)$ and i from $G^{\prime}$ and $I^{\prime}$ respectively.

- Step 2: Repeat Step 1 until the procedure is terminated.

The procedure in theorem 3 essentially determines allocation for the bidders in decreasing order of their budgets. The bidder with the highest budget goes first and gets the maximally relevant bundle from $G$. The bidder with the next highest budget goes next and gets the maximally relevant bundle from the remaining goods, and so on until all bidders have taken their turn or there are no more goods remaining. Note that a bidder with a relatively high budget may get the empty bundle if, when it is his turn, the remaining goods are all worthless to him. Also, the seller has zero budget and goes last, so he would keep all goods left over when all bidders have taken their turns. 
The procedure in theorem 3 is said to reach bidder $i$ when it is bidder $i$ 's turn to get his goods. It is obvious that if bidder $i$ has budget higher than bidder $j$, then the procedure reaches bidder $i$ before bidder $j$. The order in which bidders are reached by the procedure is referred to as the order of allocation.

PROOF. The first iteration of step 1 is established by applying theorem 2 to the isolated group $(I, G, V)$. I now show the second iteration of step 1 . Let $G^{\prime}$ and $I^{\prime}$ be the remaining goods and bidders after the first iteration. Label the bidder with the highest budget in $I^{\prime}$ bidder 1 . Consider 2 cases: $V_{1}\left(G^{\prime}\right)=\emptyset$ and $V_{1}\left(G^{\prime}\right) \neq \emptyset$.

In the case where $V_{1}\left(G^{\prime}\right)=\emptyset$, none of the bundles from $G^{\prime}$ is valuable to bidder 1 , so by non-wastefulness bidder 1 must get the empty bundle.

In the case where $V_{1}\left(G^{\prime}\right) \neq \emptyset$, consider the value sets $\hat{V}_{I^{\prime}}$ such that $\left(I^{\prime}, G^{\prime}, \hat{V}\right)$ is an isolated rectangular group. By theorem 2, bidder 1 wins $\hat{V}_{1}\left(G^{\prime}\right)=G^{\prime}$.

Now consider the change in value set for a bidder $i \in I^{\prime}, i \neq 1$ from $\hat{V}_{i}$ to $V_{i}$. Because the first iteration of step 1 is true, only the goods in $G^{\prime}$ are available to $i$, so he can only win goods in $G^{\prime}$. If he wins some good in $G^{\prime}$, then when his value set is $\hat{V}_{i}$ he has a profitable deviation: report $V_{i}$ instead. Because this violates IC, bidder $i$ must still be losing when his reported value set is $V_{i}$. Non-bossiness implies that bidder 1 still wins all the goods in $G^{\prime}$ after this change. By this reasoning, the allocation $\phi_{1}^{a}=G^{\prime}$ is preserved if I apply the change from $\hat{V}_{i}$ to $V_{i}$ for each bidder $i \in I^{\prime}, i \neq 1$, sequentially bidder by bidder. Hence, when value sets are $V_{i}$ for $i \in I^{\prime}, i \neq 1$ and $\hat{V}_{1}$ for bidder 1, bidder 1 wins all the goods in $G^{\prime}$.

Finally, consider the allocation when value sets are $\left(V_{i}\right)_{i \in I}$. Because the first iteration of step 1 is true, bidder 1 can only win goods in $G^{\prime}$. If bidder 1 does not win a bundle containing $V_{1}\left(G^{\prime}\right)$ then he has a profitable deviation: report value set $\hat{V}_{1}$ instead. By the previous paragraph, such a report will win him all the goods in $G^{\prime}$ which is always profitable by greediness. Therefore, bidder 1 wins a bundle containing $V_{1}\left(G^{\prime}\right)$. By non-wastefulness, he wins exactly $V_{1}\left(G^{\prime}\right)$.

Other iterations of step 1 can be established using the same argument above.

While theorem 3 completely pins down the allocation, it is silent on the payments. As can be expected, the payments are used to keep the bidders honest about their budgets. The intuition can be obtained from the case of isolated rectangular groups. In such a group, the bidder with the highest budget wins all goods. If he reports his budget to be anything less than the second highest budget, then he no longer has the highest budget and is therefore losing. Therefore he must pay the second highest budget. In general, a bidder $i$ winning the bundle $x_{i}$ pays the highest budget among the bidders who would win, in bidder $i$ 's absence, a bundle overlapping $x_{i}$. In a sense, bidder $i$ is paying for the highest "externality" that his participation imposes on other bidders.

Theorem 4. Let $(u, b)$ be given. The allocation $\phi^{a}(u, b)$ is given by theorem 3. The payment $\phi_{i}^{p}(u, b)$ of bidder $i$ is given by the following procedure:

- Step 1: Compute $\phi^{a}\left(u_{-i}, b_{-i}\right)$, the allocation for the profile where bidder $i$ is excluded, using the procedure in theorem 3.

- Step 2: Let $J=\left\{j: \phi_{j}^{a}\left(u_{-i}, b_{-i}\right) \cap \phi_{i}^{a}(u, b) \neq \emptyset\right\}$ be the set of bidders whose hypothetical allocation in the absence of bidder $i$ overlap with $i$ 's allocation. Let $p_{i}=\max _{k \in J} b_{k}$ be the highest budget among such bidders.

- Step 3: Bidder i's payment is $\phi_{i}^{p}(u, b)=p_{i}$. 
Proof. The allocation rule $\phi^{a}(u, b)$ is given by theorem 3. The payment rule shall be shown using the allocation rule. I show that if $\phi_{i}^{p}(u, b) \neq p_{i}$ for bidder $i$ then there is profitable deviation for bidder $i$. Label bidders at profile $(u, b)$ in decreasing order of budget and suppose the bidder with budget $p_{i}$ is bidder $k$, i.e, $b_{k}=p_{i}$. The order of allocation is then $(1,2, \ldots, i-1, i, i+1, \ldots, k-1, k, k+1, \ldots)$. Denote by $G_{j}$ the set of goods remaining when the allocation procedure reaches a bidder $j$. For the profile $\left(u_{-i}, b_{-i}\right)$ where $i$ is excluded, the order of allocation is $(1,2, \ldots, i-1, i+1, \ldots, k-1, k, k+1, \ldots)$. Denote by $\hat{G}_{j}$ the set of goods remaining when the allocation procedure, implemented without bidder $i$, reaches bidder $j$. By definition of the allocation procedure, $\phi_{i}^{a}(u, b)=V_{i}\left(G_{i}\right), \phi_{j}^{a}\left(u_{-i}, b_{-i}\right)=V_{j}\left(\hat{G}_{j}\right)$, and by definition of $k$, $V_{j}\left(\hat{G}_{j}\right) \cap V_{i}\left(G_{i}\right)=\emptyset$ for all $j<k$ and $V_{k}\left(\hat{G}_{k}\right) \cap V_{i}\left(G_{i}\right) \neq \emptyset$

Suppose $\phi_{i}^{p}(u, b)>b_{k}$. Consider a budget report $\hat{b}_{i}=b_{k}+\varepsilon<\phi_{i}^{p}(u, b)$ for small $\varepsilon>0$ so that the order of allocation at the profile $\left(u, b_{-i}, \hat{b}_{i}\right)$ would be $(1,2, \ldots, i-1, i+1, \ldots, k-1, i, k, k+1, \ldots)$, i.e., bidder $i$ would be immediately before bidder $k$. When the allocation procedure reaches bidder $i$, the set of remaining goods must be $\hat{G}_{k}$. Because $V_{j}\left(\hat{G}_{j}\right) \cap V_{i}\left(G_{i}\right)=\emptyset$ for all $j<k$, the set $V_{i}\left(G_{i}\right)$ is still available, so $i$ wins at least $V_{i}\left(G_{i}\right)$. Individual rationality means that $i$ does not pay more than $\hat{b}_{i}$, so reporting $\hat{b}_{i}$ can win $i$ the same bundle at a lower price and is therefore a profitable deviation.

Suppose $\phi_{i}^{p}(u, b)<b_{k}$. Consider a valuation report $\hat{u}_{i}$ with value set $\hat{V}_{i}=\left\{V_{i}\left(G_{i}\right)\right\}$ and a budget report $\hat{b}_{i}=b_{k}-\varepsilon>\phi_{i}^{p}(u, b)$ for some $\varepsilon>0$ so that the order of allocation is now $(1,2, \ldots, i-1, i+1, \ldots, k-$ $1, k, i, k+1, \ldots)$ i.e., bidder $i$ would be immediately after bidder $k$. At this report, bidder $i$ must still win $V_{i}\left(G_{i}\right)$ because otherwise, a bidder with value set $\hat{V}_{i}$ and budget $\hat{b}_{i}$ has a profitable deviation: report $V_{i}$ and $b_{i}$ instead. But then the allocation of bidder $i$ at this profile $\left(u_{-i}, \hat{u}_{i}, b_{-i}, \hat{b}_{i}\right)$ contradicts the allocation rule. Given the order of allocation, at bidder $k$ 's turn, the set $\hat{G}_{k}$ is available, so $k$ wins $V_{k}\left(\hat{G}_{k}\right)$. Since $V_{k}\left(\hat{G}_{k}\right) \cap V_{i}\left(G_{i}\right) \neq \emptyset$, bidder $i$ cannot win $V_{i}\left(G_{i}\right)$.

The intuition is that if bidder $i$ has to pay more than $b_{k}$, he can misreport his budget to be arbitrarily close to but higher than $b_{k}$. The allocation rule ensures that he still gets the same bundle as before. If a bidder $i$ has to pay less than $b_{k}$, he would still win the same bundle if he reported a budget less than $b_{k}$, but that would contradict the allocation rule.

The procedure to produce allocation and payment in theorem 4 is referred to as the Iterative Second Price Auction (ISPA). As an illustration, consider the profile in table 9. A bundle in a bidder's value set are represented by a rectangle $\times$ or $\checkmark$ stretching over the goods contained in the bundle. The ISPA procedure leads to allocation marked by rectangles $\checkmark$. For example, bidder 1 has the highest budget, so he gets his maximally relevant bundle $\left\{g_{1}, g_{2}\right\}$. Next, bidder 2 gets $\left\{g_{5}\right\}$, the only relevant bundle out of the remaining goods. Next, bidder 3 gets his maximally relevant bundle out of the remaining goods, $\left\{g_{3}, g_{4}\right\}$. Bidders 4 and 5 are losing bidders. To calculate payment for bidder 1, consider what would happen if bidder 1 were absent. Bidder 2 would win $\left\{g_{2}, g_{3}, g_{5}\right\}$ and bidder 4 would win $g_{1}$. Because bidder 1's allocation is causing externality on bidder 2 and bidder 4 , and bidder 2 has higher budget than bidder 4 , bidder 1 pays bidder 2's budget, which is 7. Other bidders' payments are similarly determined.

Theorem 4 states that ISPA is "necessary" for any mechanism that is incentive compatible, individually rational, symmetric, non-bossy and non-wasteful - any other outcome rule would lead to violation of some of the criteria. The question remains whether ISPA is "sufficient," i.e., whether it satisfies all five criteria. The answer is in the affirmative. Before I proceed to state and prove the result, it is necessary to note that ISPA can determine the outcome for the greedy and generic domain only. Incentive compatibility requires that no bidder is strictly better off by misreporting, even if that misreport creates a profile that is nongeneric. ISPA is, however, completely silent on the outcome at generic profiles. Fortunately, it is easy to attain incentive compatibility once ISPA is augmented with a tie-breaking rule that enables it to handle nongeneric profiles. A tie-breaking rule is an exogenous deterministic indexing of the bidders that determines, 
Table 9: Illustration of ISPA

\begin{tabular}{|c|c|c|c|c|c|c|c|}
\hline & \multicolumn{5}{|c|}{ Goods } & \multirow[t]{2}{*}{ Budget } & \multirow[t]{2}{*}{ Payment } \\
\hline & $g_{1}$ & $g_{2}$ & $g_{3}$ & $g_{4}$ & $g_{5}$ & & \\
\hline 1 & $\checkmark$ & $\checkmark$ & & & & 10 & 7 \\
\hline 2 & & & & & $\checkmark$ & 7 & 4 \\
\hline 3 & & & & & & 6 & 2 \\
\hline 4 & $x$ & & & & $\times$ & 4 & 0 \\
\hline 5 & $x$ & $x$ & $x$ & $\times$ & & 2 & 0 \\
\hline
\end{tabular}

in conjuction with the magnitudes of budgets, the order of allocation. Augmented ISPA is ISPA with the additional provision that if at any step in the ISPA procedures there are two or more bidders with the highest budget among the remaining bidders, then the order of allocation is determined by the exogenous indices of the bidders, in increasing order, i.e., the bidder with the lower index goes first.

Theorem 5. Augmented ISPA is incentive compatible, individually rational, symmetric, non-wasteful and non-bossy.

PROOF. It is easy to see that augmented ISPA is individually rational, symmetric and non-wasteful and non-bossy. I now prove incentive compatibility by showing that there is no profitable deviation from any generic profile. I write $\varphi_{i}^{a}(\cdot)$ and $\varphi_{i}^{p}(\cdot)$ to mean the allocation and payment for bidder $i$ by the augmented ISPA mechanism.

Consider a bidder $i$ with true valuation and budget $\left(u_{i}, b_{i}\right)$, and any report by other bidders $\left(u_{-i}, b_{-i}\right)$. Label the bidders at profile $(u, b)$ in decreasing order of budget and suppose the bidder with the budget $\varphi_{i}^{p}(u, b)$ is bidder $k$, i.e., $b_{k}=\varphi_{i}^{p}(u, b)$. The order of allocation is then $(1,2, \ldots, i-1, i, i+1, \ldots, k-1, k, k+$ $1, \ldots)$. Denote by $G_{j}$ the set of goods remaining when the allocation procedure reaches a bidder $j$. By ISPA allocation procedure, $\varphi_{j}^{a}(u, b)=V_{j}\left(G_{j}\right)$. For the profile $\left(u_{-i}, b_{-i}\right)$ where bidder $i$ is excluded, the order of allocation is $(1,2, \ldots, i-1, i+1, \ldots, k-1, k, k+1, \ldots)$. Denote by $\hat{G}_{j}$ the set of goods remaining when the allocation procedure, implemented without bidder $i$, reaches bidder $j$. By ISPA allocation procedure, $\varphi_{j}^{a}\left(u_{-i}, b_{-i}\right)=V_{j}\left(\hat{G}_{j}\right)$. Note that for bidders $h<i$, their available sets and allocation are unaffected by bidder $i$ 's presence, i.e., $G_{h}=\hat{G}_{h}$ and so $V_{h}\left(G_{h}\right)=V_{h}\left(\hat{G}_{h}\right)$ which implies $\varphi_{h}^{a}(u, b)=\varphi_{h}^{a}\left(u_{-i}, b_{-i}\right)$.

Consider any budget report $\hat{b}_{i}$ by bidder $i$. If the $\hat{b}_{i}$ is such that the order of allocation, taking into account the tie-breaking rule, is unchanged, then when the procedure reaches bidder $i$, the set $G_{i}$ is still available. By greediness, it is optimal, conditional on the report $\hat{b}_{i}$, for $i$ to win $V_{i}\left(G_{i}\right)$. Given that $i$ is winning $V_{i}\left(G_{i}\right)$, the payment must be $b_{k}$. This outcome for bidder $i$ is the same as the outcome arising from truthful reporting.

Suppose the report $\hat{b}_{i}$ is such that $i$ 's turn, taking into account the tie-breaking rule, is shifted up, i.e., the order of allocation is changed to, say, $(1,2, \ldots, j-1, i, j, j+1, \ldots, i-1, i+1, \ldots, k-1, k, k+1, \ldots)$. When it is $i$ 's turn, the set $G_{j}$ is available. If $i$ 's valuation report $\hat{u}_{i}$ is such that $i$ wins some good from $V_{j}\left(G_{j}\right)$, which is also $j$ 's allocation with $i$ excluded $\varphi_{j}^{a}\left(u_{-i}, b_{-i}\right)$, then $\varphi_{i}^{a}\left(u_{-i}, \hat{u}_{i}, b_{-i}, \hat{b}_{i}\right) \cap \varphi_{j}^{a}\left(u_{-i}, b_{-i}\right) \neq \emptyset$. Because $i$ is causing externality on $j, i$ must pay at least $b_{j}$, which exceeds $i$ 's actual budget $b_{i}$. So any good in $V_{j}\left(G_{j}\right)$ is unaffordable to $i$. Similarly, any good in $V_{h}\left(G_{h}\right)$ is unaffordable to $i$ for any $h<i$. Therefore, though the set $G_{j}$ is available to $i$, only goods from the set $G_{j}-V_{j}\left(G_{j}\right)-V_{j+1}\left(G_{j+1}\right)-\ldots-V_{i-1}\left(G_{i-1}\right)=G_{i}$ are affordable to $i$. Greediness implies that it is optimal, conditional on the report $\hat{b}_{i}$, for $i$ to win $V_{i}\left(G_{i}\right)$. The price must then be $b_{k}$. This outcome is again the same as the outcome resulting from truthful reporting. 
Suppose the report $\hat{b}_{i}$ is such that $i$ 's turn, taking into account the tie-breaking rule, is shifted down to between $i+1$ and $k$, i.e., the order of allocation is now, say, $(1,2, \ldots, i-1, i+1, \ldots, j-1, i, j, j+1, . ., k, k+$ $1, \ldots)$ then the set of goods available to $i$ is $\hat{G}_{j}$. By definition of ISPA, $\hat{G}_{j} \subseteq \hat{G}_{j-1} \subseteq \ldots \subseteq \hat{G}_{i+1}$. However, note that $\hat{G}_{i+1}=G_{i}$, so $\hat{G}_{j} \subseteq G_{i}$. Because $V_{h}\left(\hat{G}_{h}\right) \cap V_{i}\left(G_{i}\right)=\emptyset$ for all $h<k$, when it is $i$ 's turn the set $V_{i}\left(G_{i}\right)$ is still available. Greediness implies that $i$ 's optimal strategy, conditional on the report $\hat{b}_{i}$, is to win $V_{i}\left(G_{i}\right)$, which will cost him $b_{k}$. Therefore, this report cannot profit bidder $i$.

Finally, suppose that $\hat{b}_{i}$ is such that $i$ 's turn, taking into account the tie-breaking rule, is shifted down to after $k$, i.e., the order of allocation is $(1,2, \ldots i-1, i+1, \ldots, k-1, k, k+1, \ldots, j-1, i, j, \ldots)$. The set of goods available to $i$ is now $\hat{G}_{j}$, which is a strict subset of $\hat{G}_{i+1}$ (because bidder $k$ wins $V_{k}\left(\hat{G}_{k}\right) \neq \emptyset$ ), which is the same as $G_{i}$. But because $k$ has his turn before $i$ and has taken $V_{k}\left(\hat{G}_{k}\right)$ which overlaps with $V_{i}\left(G_{i}\right)$, the bundle $V_{i}\left(G_{i}\right)$ is no longer available. Therefore, $i$ can only win $V_{i}\left(\hat{G}_{j}\right) \neq V_{i}\left(G_{i}\right)$. By definition of greediness, $u_{i}\left(V_{i}\left(\hat{G}_{j}\right)\right)<u_{i}\left(V_{i}\left(G_{i}\right)\right)-b_{i}$, so bidder $i$ must be worse off compared to truthful reporting.

Table 9 can provide some intuition for the incentive compatibility of ISPA and also illustrates the role of greediness. Suppose bidder 1 reports his budget as 6.9 instead of 10. In such deviation, bidder 1 would win $g_{1}$ and pay 4. So, his payoff from the deviation is $u(\{1\})-4$, whereas it was $u(\{1,2\})-7$ in the original allocation. Since bidder 1 is greedy, $u(\{1,2\})-u(\{1\})>10$, and so $u(\{1,2\})-7>u(\{1\})-4$. Therefore, this misreport does not benefit bidder 1 .

A few remarks are in order. Recall that there are two conditions for greediness. The first requires the maximally relevant bundle from any set of goods to be unique, and the second requires any increase in valuation to exceed budget. It is worth noting that ISPA can also accommodate preferences that satisfy the second condition but not the first. For such preferences, uniqueness of the maximally relevant bundle can be artificially satisfied through the use of an exogenous tie-breaking rule. For example, one could index all the goods, create a lexicographic order over the bundles based on the index and pick the maximally relevant bundle that is lexicographically smallest. With such a tie-breaking rule, these preferences become effectively greedy, and ISPA can satisfy the five criteria.

It is of interest whether the results for the greedy domain hold if a standard definition of non-bossiness is used. In the standard definition, if a bidder's report does not change his own allocation, then it cannot change other bidders' allocation, regardless of whether the bidder is winning or losing. Since the standard definition is stronger than my definition, the "necessity" of ISPA (theorem 4) still holds. To show that ISPA indeed satisfies the standard notion of non-bossiness (theorem 5), I need to show that if bidder $i$ 's report does not change his own allocation, then it does not change any of the other bidders' allocations either. But this is obvious. If bidder $i$ 's allocation is unchanged, then the set of goods collectively available to the other bidders is also unchanged. The order of allocation for the other bidders is unchanged as well because their budgets have not changed. So the set of goods available to a bidder as augmented ISPA reaches that bidder is unchanged. Therefore, the allocation of the other bidders must be unchanged.

There are many possible tie-breaking rules, so there are correspondingly many possible augmented ISPA mechanisms. However, the outcome of all of these mechanisms agree on the domain of greedy generic profiles, as shown by theorem 4. In this sense, one can think of ISPA as the unique mechanism satisfying the five criteria in the greedy generic domain. The problem for the greedy domain is now effectively solved. The allocation rule is remarkably simple, though this simplicity is a consequence of the greediness of the bidders. Given the allocation rule, the principle of having a bidder pay for his externality leads to the payment rule that provides the right incentives for truthful reporting. The characterization of mechanisms satisfying the five criteria is completed: there exists only one such mechanism on generic profiles, and its outcome is given by ISPA. 


\section{Impossibility result for the general domain}

Given the positive result for the greedy domain, one might hope that there exists a mechanism satisfying the five criteria for the general domain as well. Unfortunately, no such mechanism exists. Before I state and prove the impossibility result, I need two lemmas which state that, given the results on the greedy domain, certain things must hold in the general domain.

Let $\Phi(\cdot)$ be a mechanism that is incentive compatible, individually rational, symmetric, non-wasteful and non-bossy for the general domain, assuming that such a mechanism exists. The payoff of the highest bidder, bidder 1, must be at least what he would get from getting the grand bundle $G$ at the price equal to the second highest budget. This is because the option of winning $G$ at the second highest budget is always available to bidder 1: bidder 1 can pretend to be greedy.

Lemma 8. Let $(u, b)$ be given and suppose $b_{1}=\max _{i \in I} b_{i}$, and $b_{2}=\max _{i \in I-1} b_{i}$. Then $v_{1}\left(\Phi_{1}(u, b)\right) \geq$ $v_{1}\left(G, b_{2}\right)$.

Proof. Consider the profile $\left(\hat{u}_{-1}, \hat{u}_{1}, b_{-1}, \hat{b}_{1}\right)$ where bidders are all greedy with value sets $\left(\hat{V}_{i}\right)_{i \in I}$ such that $(I, G, \hat{V})$ is a isolated rectangular group. Budgets of all bidders other than bidder 1 are the same as profile $(u, b)$. Bidder 1's budget is $\hat{b}_{1}=b_{2}+\varepsilon$ for some small $\varepsilon>0$. By theorem 1, bidder 1 wins $G$ and all other bidders are losing.

Now consider the change from $\hat{u}_{j}$ to $u_{j}$ for a bidder $j \neq 1$. Bidder $j$ must remaining losing, otherwise when $j$ 's valuation is $\hat{u}_{j}$ he has a profitable deviation: report $u_{j}$ instead. Non-bossiness means that bidder 1 still wins $G$. This reasoning allows me to change valuation reports of all bidders $j \neq 1$, bidder by bidder, from $\hat{u}_{j}$ to $u_{j}$ without changing the allocation of bidder 1 . So at the profile $\left(u_{-1}, \hat{u}_{1}, b_{-1}, \hat{b}_{1}\right)$ bidder 1 wins $G$. Individual rationality implies that he pays at most $\hat{b}_{1}$, which can be set arbitrarily close to $b_{2}$.

Since the outcome $\left(G, b_{2}\right)$ is available to bidder 1 , his payoff is bounded below by $v_{1}\left(G, b_{2}\right)$.

It has been established that if all bidders are greedy and the bidder wins the highest budget wins all the goods in $G$, then he must pay the second highest budget. This is also true if the highest budget bidder is not greedy: if he wins all goods he must pay the second highest budget.

Lemma 9. Let $(u, b)$ be given such that bidder 1 has the highest budget, and that all other bidders are greedy. Let $b_{2}=\max _{i \in I-1} b_{i}$ be the second highest budget. If $\Phi_{1}^{a}(u, b)=G$ then $\Phi_{1}^{p}(u, b)=b_{2}$.

Proof. Consider the profile $\left(u_{-1}, \hat{u}_{1}, b\right)$ where all bidders, including bidder 1 , are greedy, and that $V_{1}(G)=$ $G$. By theorem 4, the outcome must coincide with ISPA outcome, in which bidder 1 wins $G$ at price $b_{2}$.

Suppose that at the profile $(u, b)$, bidder 1 wins $G$ but at a price lower than $b_{2}$. Then when bidder 1's valuation is $\hat{u}_{1}$ he has a profitable deviation: report $u_{1}$ instead. If at profile $(u, b)$ bidder 1 wins $G$ at a price higher than $b_{2}$ then bidder 1 has a profitable deviation: report $\hat{u}_{1}$ instead.

Therefore, if bidder 1 wins $G$, he must pay the second highest budget $b_{2}$, even if he is not greedy.

Theorem 6. For the general domain there is no mechanism that is incentive compatible, individually rational, symmetric, non-wasteful and non-bossy.

Proof. Suppose in negation that $\Phi(\cdot)$ is a mechanism that is incentive compatible, individually rational, symmetric, non-wasteful and non-bossy for the general domain. This proof makes use of tables to illustrate a counter-example, by showing that certain allocations and payments must happen at certain profiles and thereby arriving at a contradiction. For example, the table under "Profile 1" in Table 10 shows a profile with two bidders, bidder 1 and bidder 2 , and 2 goods, $A$ and $B$, the bidders' valuations and budgets $(b)$. The 
Table 10: A counter example

\begin{tabular}{c|ccc|c|c|c}
\multicolumn{8}{c}{ Profile 1 } \\
\hline & \multicolumn{7}{c}{ Bundles } & $b$ & $p$ & $v$ \\
& A & B & AB & & & \\
\hline 1 & 12 & 1 & 13 & 7 & 3 & 10 \\
2 & 10 & 4 & 14 & 3 & 0 & 0
\end{tabular}

\begin{tabular}{c|ccc|c}
\multicolumn{4}{c}{ Profile 2 } \\
\hline & \multicolumn{3}{|c}{ Bundles } & $b$ \\
& A & B & AB & \\
\hline 1 & 12 & 1 & 13 & 2 \\
2 & 10 & 4 & 14 & 3
\end{tabular}

\begin{tabular}{|c|c|c|c|c|}
\hline \multicolumn{5}{|c|}{ Profile 3} \\
\hline & \multicolumn{3}{|c|}{ Bundles } & $b$ \\
\hline & A & B & $\mathrm{AB}$ & \\
\hline 1 & 12 & 1 & 13 & 7 \\
\hline 2 & 0 & 4 & 4 & 3 \\
\hline
\end{tabular}

\begin{tabular}{c|ccc|c|c|c}
\multicolumn{7}{c}{ Profile 4 } \\
\hline & \multicolumn{3}{|c|}{ Bundles } & $b$ & $p$ & $v$ \\
& A & B & AB & & & \\
\hline 1 & 12 & 0 & 12 & 7 & 0 & 12 \\
2 & 0 & 4 & 4 & 3 & 0 & 4
\end{tabular}

allocation is indicated by having a box around the number representing a bidder's valuation for the allocated bundle. Payment $(p)$ and payoff $(v)$ are listed to the right. The table shows that bidder 1 has budget of 7 and wins the bundle $\{A, B\}$, which is worth 13 to him, at the price of 3 . Note that payments or payoffs are omitted from the tables when not necessary for the proof.

Consider profile 1 in Table 10. By lemma 8, bidder 1's payoff is at least $13-3=10$, so he must win either $\{A\}$ or $\{A, B\}$. If he wins $\{A\}$, then because $\{A\}$ yields valuation of 12 his payment is at most 2 . Incentive compatibility guarantees that he must still be winning if he reports a budget of 2 . In other words, bidder 1 in profile 2 must be winning (otherwise bidder 1 in profile 2 can profitably deviate to profile 1). However, at profile 2, lemma 8 implies that bidder 2's payoff is at least $14-2=12$, dictating that bidder 2 wins $\{A, B\}$ and bidder 1 wins nothing - a contradiction. Therefore, at profile 1 bidder 1 must win $\{A, B\}$, and, by lemma 9 and greediness of bidder 2, pay 3. This outcome is indicated in the table.

Similar reasoning applies to profile 3 . By lemma 8 , bidder 1's payoff is at least $13-3=10$, so bidder 1 wins either $\{A\}$ or $\{A, B\}$. If bidder 1 wins $\{A, B\}$, then because bidder 2 is greedy, by lemma 9 , bidder 1 has to pay 3 and get a payoff of 10 . Consider the deviation by bidder 1 to profile 4 . Because profile 4 is a greedy profile, the outcome is determined by ISPA, as shown in the table. Note that bidder 1 wins $\{A\}$ and pays nothing, getting a payoff of 12 , an improvement over truthful reporting, violating incentive compatibility. Therefore, at profile 3, bidder 1 wins $\{A\}$ and, by non-wastefulness, bidder 2 wins $\{B\}$.

Now consider profile 1 and profile 3 . Given the outcome at these profiles, bidder 2 at profile 1 can profitably deviate to profile 3 and win $\{B\}$ for the price of at most 3 , violating incentive compatibility.

Since at profile 1 there is incentive to misreport, $\Phi(\cdot)$ is not incentive compatible.

Though there may be many reasons why no mechanism exists for the general domain, one can get some intuition from the ISPA mechanism for the greedy domain, as well as the generalized Vickrey-ClarkesGroves (VCG) mechanism for the domain where bidders are not budget-constrained. In both mechanisms (applied in their respective domains), each bidder essentially faces a personalized price for each bundle which comes from the externality he causes on other bidders by taking that bundle. More importantly, the prices align incentives in these environments, in the sense that given these prices the bidders' optimal bundles do not overlap with one another. In other words, there is no incentive to manipulate prices. Such prices can, in theory, be constructed for the general domain using the externality principle as well. However, when these prices are such that the bidders' choices are not congruent, a bidder can have incentive to manipulate these prices. Because prices facing a bidder $i$ are determined by other bidders, they are exogenous to bidder 
$i$ and cannot be manipulated by bidder $i$. However, bidder $i$ can manipulate prices facing other bidders, thus inducing them to choose different bundles. The manipulation by bidder 2 in profile 1 in the counter example can be thought of as effectively reducing the price for bidder 1 of $\operatorname{good} A$, thereby inducing bidder 1 to "choose" $\{A\}$ instead of $\{A, B\}$.

\section{Conclusion}

In this paper I consider the problem of combinatorial auction where bidders have budget constraints . I look for deterministic mechanisms satisfying five criteria: incentive compatibility, individual rationality, symmetry, non-wastefulness and non-bossiness. I show that for the greedy domain, one unique mechanism exists: the Iterative Second Price Auction. However, for the general domain, there is no such mechanism.

The negative result for the general domain has several implications. If the five criteria must be met, the auctioneer has to find a way to eliminate budget constraints of the bidders, through financing schemes or other means. If budget constraints are a unavoidable, then some criteria must be relaxed. For example, ex-post incentive compatibility is a particularly strong requirement - it could be relaxed to either ex-ante or interim incentive compatibility. However, the analysis of mechanisms that are ex-ante or interim incentive compatible is in general complicated by the multi-dimensionality of the distribution of types. There are wasteful mechanisms that have good incentive properties and can accommodate budget constraints, such as the Deferred Acceptance Auction by Segal and Milgrom (2014). Non-bossiness can also be relaxed. From a practical point of view, allowing losing bidders to change the allocation of winning bidders while staying losing may seem strange for auction participants, but the larger space of such mechanisms may contain auction formats that are theoretically interesting.

Another approach is to look for randomized mechanisms. In a randomized mechanism, the outcome for each bidder can be a probability distribution over deterministic outcomes. Randomized mechanisms have been used in algorithmic mechanism design (Dughmi and Vondrak, 2011) and it would be interesting to see if they can accommodate budget constraints.

\section{References}

Ausubel LM (2004) An efficient ascending-bid auction for multiple objects. American Economic Review 94(5):1452-1475

Bhattacharya S, Conitze V, Munagala K, Xia L (2010) Incentive compatible budget elicitation in multi-unit auctions. In: In Proceedings of the Annual AM-SIAM Symposium on Disrete Algorithms, pp 554-572

Borgs C, Chayes J, Immorlica N, Mahdian M, Saberi A (2005) Multi-unit auctions with budget-constrained bidders. In: EC 05: Proceedings of the 6th ACM conference on Electronic commerce, pp 44-51

Bulow J, Levin J, Milgrom P (2009) Winning play in spectrum auctions. NBER Working Paper

Che YK, Gale I (1996) Expected revenue of all-pay auctions and first-price sealed-bid auctions with budget constraints. Economic Letters 50:373-379

Che YK, Gale I (2000) The optimal mechanism for selling to a budget-constrained buyer. Journal of Economic Theory 92:1982000

Day R, Milgrom P (2008) Core-selecting package auctions. International Journal of Game Theory 36:393-440

Dobzinski S, Lavi R, Nisan N (2008) Multi-unit auctions with budget limits. FOCS

Dughmi S, Vondrak J (2011) Limitations of randomized mechanisms for combinatorial auctions. FOCS pp 502-511

Fiat A, Leonardi S, Saia J, Sankowski P (2011) Single valued combinatorial auctions with budgets. ACM Conference on Electronic Commerce

Gagan Goel RPL Vahab Mirrokni (2012) Polyhedral clinching auctions and the adwords polytope. In: STOC '12 Proceedings of the forty-fourth annual ACM symposium on the Theory of Computing, ACM New York, pp 107-122

Hafalir IE, Ravi R, Sayedi A (2011) A near pareto optimal auction with budget constraints. Games and Economic Behavior 74:699708

Laffront JJ, Robert J (1996) Optimal auction with financially constrained buyers. Economic Letters 52(2):181-186 
Milgrom P (2000) Putting auction theory to work: The simultaneous ascending auction. Journal of Political Economy 108(2):245272

Nisan N, Ronen A (2007) Computationally feasible vcg mechanisms. Journal of Artificial Intelligence Research 29:19-47

R Lavi MM (2011) A note on the incompatibility of strategy-proofness and pareto-optimality in quasi-linear settings with public budgets. In: Internet and Network Economics, Lecture Notes in Computer Science, Springer Berlin Heidelberg, p 417

Satterthwaite MA, Sonnenschein H (1981) Strategy-proof allocation mechanisms at differentiable points. The Review of Economic Studies 48(4):587-597

Segal I, Milgrom P (2014) Deferred acceptance auctions and radio spectrum reallocation, mimeo

\section{A. Appendix}

\section{A.1. Proof of lemma 7}

Suppose in negation that $b_{i}>b_{j}$ and $\phi_{j}^{a}(u, b)=x_{j} \in V_{i}$ but that $\phi_{i}^{a}(u, b)=\emptyset$. Suppose $i$ shrinks his valued set to $\hat{V}_{i}=\left\{x_{j}\right\}$. By lemma 3, $i$ is still losing. By non-bossiness, bidder $i$ 's change in report cannot change bidder $j$ 's allocation, so bidder $j$ still wins $x_{j}$. Suppose now $j$ also shrinks his value set to $\hat{V}_{j}=\left\{x_{j}\right\}$. By corollary $2, j$ must still win $x_{j}$. So at the new modified profile where $\hat{V}_{i}=\hat{V}_{j}=\left\{x_{j}\right\}, j$ wins $x_{j}$ and $i$ is losing. This holds true too even when $u_{i}\left(x_{j}\right)=u_{j}\left(x_{j}\right)$, i.e., when $x_{j}$ yields the same valuation to both bidders.

Denote the profile obtained after the above changes by $(\hat{u}, b)$. Individual rationality implies that $p_{j} \leq b_{j}$. Suppose now that bidder $j$ increases his budget to be $\hat{b}_{j}=b_{i}$ (note that $u_{i}\left(x_{j}\right)$ and $u_{j}\left(x_{j}\right)$ can be chosen to be large enough to ensure that with this new budget bidder $j$ is still greedy). By lemma 6 , bidder $j$ must still win $x_{j}$. Suppose now that bidder $i$ lowers his budget to be $\hat{b}_{i}=b_{j}$. Lemma 6 implies he is still losing.

Effectively, bidders $i$ and $j$ have "swapped" their budgets and valuations while preserving their allocation bundles, contradicting symmetry which requires that their allocation bundles must be swapped also.

\section{A.2. Proof of theorem 1}

In this proof, I restrict attention to isolated groups. I use the term value set basis to refer to the set of goods whose non-empty subsets are elements of the value set. A value set is covered by a value set basis if it contains exactly all non-empty subsets of the value set basis.

Definition 12. A value set $V_{i}$ is covered by a value set basis $V_{i}^{b}$ if $V_{i}^{b} \subseteq G$ and $V_{i}=2^{V_{i}^{b}}-\emptyset$.

A rectangular isolated group can be thought of as a profile where each bidder's value set basis is simply $G$. It will be useful to have a way of referring to groups of certain shapes like triangles or squares. I use a notion that encompasses all these shapes.

Definition 13. Let $n, g, s, k$ be non-negative natural numbers. A group $\left(I, G,\left(V_{i}\right)_{i \in I}\right)$ is called $(n, g, s, k)$-shape if

- There are $n$ bidders in $I$, labeled such that $b_{1}>b_{2}>\ldots>b_{n}$.

- There are $g$ goods in $G$.

- The value set $V_{i}$ of each bidder $i$ is covered by value set basis $V_{i}^{b}$.

- $V_{1}^{b} \supseteq V_{2}^{b} \supseteq \ldots \supseteq V_{n}^{b}$.

- $1 \leq s<n$.

- For $i=1, \ldots, s,\left|V_{i}^{b}\right|=|G|$.

- For $s \leq i<n, i \neq k,\left|V_{i}^{b}-V_{i+1}^{b}\right|=1$.

- If $k \neq 0$, then $s \leq k<n$, and $\left|V_{k}^{b}-V_{k+1}^{b}\right|=2$. 
Table 11: Illustration of the "shape" of a profile

Profile 1

\begin{tabular}{|c|c|c|c|c|}
\hline & \multicolumn{4}{|c|}{$(4,3,2,3)$-shape } \\
\hline & $g_{4}$ & $g_{3}$ & $g_{2}$ & $g_{1}$ \\
\hline 1 & & $x$ & $x$ & $x$ \\
\hline 2 & & $x$ & $x$ & $x$ \\
\hline 3 & & & $x$ & $x$ \\
\hline 4 & & & & \\
\hline
\end{tabular}

Profile 2

$(4,4,4,0)$-shape

\begin{tabular}{|c|c|c|c|c|}
\hline & $g_{4}$ & $g_{3}$ & $g_{2}$ & $g_{1}$ \\
\hline 1 & $\times$ & $\times$ & $x$ & $\times$ \\
\hline 2 & $\times$ & $\times$ & $x$ & $x$ \\
\hline 3 & $\times$ & $\times$ & $\times$ & $\times$ \\
\hline 4 & $\times$ & $\times$ & $x$ & $x$ \\
\hline
\end{tabular}

Profile 3

$(4,4,1,0)$-shape

\begin{tabular}{|c|c|c|c|c|}
\hline & $g_{4}$ & $g_{3}$ & $g_{2}$ & $g_{1}$ \\
\hline 1 & $x$ & $x$ & $x$ & $\times$ \\
\hline 2 & & $x$ & $\times$ & $\times$ \\
\hline 3 & & & $\times$ & $\times$ \\
\hline 4 & & & & $x$ \\
\hline
\end{tabular}

In a $(n, g, s, k)$-shape group, value sets bases of the first $s$ bidders (indexed by budget size, in decreasing order) contain all individual goods, and but they start to "cascade" from bidder $s$ : each bidder's value set basis contains all but one good from the previous bidder's value set basis. There is an exception at bidder $k$, where the bidder $k+1$ 's value set basis contains all but two goods from bidder $k$ 's value set basis. Table 11 show profiles where $\times$ indicate that a good is in a bidder's value set basis. For example, a $(4,3,2,3)$-shape group is shown in profile 1 . When $k=0$, the decrease in size of value set basis is at most one. The profile 2 illustrates a "square" $(4,4,4,0)$-shape group, and profile 3 represents a "triangular" $(4,4,1,0)$-shape group.

I first show that at any "triangular" profile of shape $(n, n, 1,0), \phi_{1}^{a}=V_{1}(G)$. This is done through induction from the smallest triangular profile of shape $(2,2,1,0)$. Once this induction is done, I proceed to "fill out" the triangular profile to obtain the shape of a square. The base case of the shape $(2,2,1,0)$ has been done in the main text. The inductive hypothesis is that the highest-budget bidder in the group wins all the goods. I shall refer to the highest bidder as bidder 1, and his allocation simply as $\phi_{1}^{a}$. Note that for a bidder $i$ whose value set $V_{i}$ is covered by $V_{i}^{b}, V_{i}(G)=V_{i}^{b}$. The inductive hypothesis: Let $n$ be given. At any $(n, n, 1,0)$-shape group $(I, G, V), \phi_{1}^{a}=G$.

I now proceed to show that at any $(n+1, n+1,1,0)$-shape group $(I, G, V), \phi_{1}^{a}=G$. Unless stated otherwise, the inductive hypothesis is always assumed in this section. Much of the proof is based on induction on the third and forth shape argument, i.e., $s$ and $k$. The essence of the argument is very much the idea of superimposing shapes to obtain new shapes while preserving the allocation rule.

I first define the notion of reducibility: a shape is reducible to another shape if two groups of the latter shape can be superimposed to form the former shape.

Definition 14. $\left(n, g, s^{\prime}, k^{\prime}\right)$ is reducible to $(n, g, s, k)$ if for any group $\left(I, G, V^{\prime}\right)$ of shape $\left(n, g, s^{\prime}, k^{\prime}\right)$ there is a bidder $i \neq 1$ and there are two goods $g_{1}, g_{2} \in V_{i}^{b}$ such that

- The group $(I, G, \hat{V})$ with value set bases $\hat{V}_{i}^{b}=V_{i}^{b}-g_{1}$ and $\hat{V}_{j}^{b}=V_{j}^{b}$ for all $j \in I, j \neq i$, is $(n, g, s, k)$-shape, and

- The group $(I, G, \tilde{V})$ with value set bases $\tilde{V}_{i}^{b}=V_{i}^{b}-g_{2}$ and $\tilde{V}_{j}^{b}=V_{j}^{b}$ for all $j \in I, j \neq i$, is $(n, g, s, k)$-shape.

Basically, $\left(n, g, s^{\prime}, k^{\prime}\right)$ is reducible to $(n, g, s, k)$ if for any $\left(n, g, s^{\prime}, k^{\prime}\right)$-shape group there are at least two ways of removing a good from the value set basis of a bidder $i \neq 1$ to attain the shape $(n, g, s, k)$. For example, in table 12, the shape in profile 1 is reducible to the shape in profile 2 by removing either of the boxes with the minus sign, - . The shape in profile 2 is in turn reducible to the shape in profile 3 by removing either of the boxes with the minus sign.

If $\left(n, g, s^{\prime}, k^{\prime}\right)$ is reducible to $(n, g, s, k)$ then $(n, g, s, k)$ is said to be expandable to $\left(n, g, s^{\prime}, k^{\prime}\right) .\left(n, g, s_{1}, k_{1}\right)$ is said to be chain-expandable to $\left(n, g, s_{m}, k_{m}\right)$ if there is a sequence of shapes $\left(n, g, s_{t}, k_{t}\right)_{t=1, \ldots, m}$ such that for all $t<m$, $\left(n, g, s_{t}, k_{t}\right)$ is expandable to $\left(n, g, s_{t+1}, k_{t+1}\right)$. The idea of superimposition is that it is possible to track allocation as one shape is expanded into another.

Remark 1. Suppose for any $(n, g, s, k)$-shape group $(I, G, V), \phi_{1}^{a}=G$. Then for any group $\left(I, G, V^{\prime}\right)$ with shape $\left(n, g, s^{\prime}, k^{\prime}\right)$ such that $(n, g, s, k)$ is chain-expandable to $\left(n, g, s^{\prime}, k^{\prime}\right), \phi_{1}^{a}=G$.

Proof. Suppose $(n, g, s, k)$ is chain-expandable to $\left(n, g, s^{\prime}, k^{\prime}\right)$. Consider the first two shapes in the chain, $(n, g, s, k)$ and $(n, g, \ddot{s}, \ddot{k})$. By definition of reducibility, for any $(n, g, \ddot{s}, \ddot{k})$-shape group $(I, G, \ddot{V})$ there is a bidder $i \neq 1$ and two 
Table 12: Illustration of reducing shapes

Profile 1

$(4,4,2,3)$-shape

\begin{tabular}{|c|c|c|c|c|}
\hline & $g_{4}$ & $g_{3}$ & $g_{2}$ & $g_{1}$ \\
\hline 1 & $\times$ & $\times$ & $x$ & $\times$ \\
\hline 2 & $\times$ & $\times$ & $x$ & $\times$ \\
\hline 3 & & - & - & $\times$ \\
\hline 4 & & & & $x$ \\
\hline
\end{tabular}

Profile 2

$(4,4,2,2)$-shape

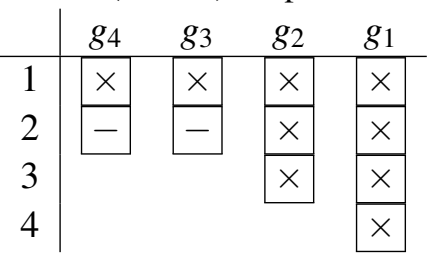

Profile 3

$(4,4,1,0)$-shape

\begin{tabular}{|c|c|c|c|c|}
\hline & $g_{4}$ & $g_{3}$ & $g_{2}$ & $g_{1}$ \\
\hline 1 & $x$ & $x$ & $x$ & $x$ \\
\hline 2 & & $x$ & $\times$ & $x$ \\
\hline 3 & & & $x$ & $\times$ \\
\hline 4 & & & & $x$ \\
\hline
\end{tabular}

groups $(I, G, \hat{V})$ and $(I, G, \tilde{V})$ of shape $(n, g, s, k)$ such that $\hat{V}_{j}^{b}=\tilde{V}_{j}^{b}=\ddot{V}_{j}^{b}$ for all $j \in I, j \neq i$ and that $\hat{V}_{i}^{b}=\ddot{V}_{i}^{b}-g_{1}$ and $\tilde{V}_{i}^{b}=\ddot{V}_{i}^{b}-g_{2}$ for some $g_{1}, g_{2} \in \ddot{V}_{i}^{b}$.

By assumption, because $(I, G, \hat{V})$ is $(n, g, s, k)$-shape, $\phi_{1}^{a}=G$, so $\phi_{i}^{a}=\emptyset$. At $(I, G, \tilde{V})$ also, $\phi_{i}^{a}=\emptyset$. Since $\ddot{V}_{i}$ is a "combination" of $\hat{V}_{i}$ and $\tilde{V}_{i}$, by lemma 4 , it must be that $\phi_{i}^{a}=\emptyset$ for $(I, G, \ddot{V})$ as well. Because bidder $i$ stays losing when his value set changes from $\tilde{V}_{i}$ to $\ddot{V}_{i}$, by non-bossiness, bidder 1's allocation is unchanged as well. Therefore, for $(I, G, \ddot{V}), \phi_{1}^{a}=G$.

So if $\phi_{1}^{a}=G$ for a group $(I, G, V)$ of a shape in the chain, then the same is true for a group $(I, G, \tilde{V})$ of the next shape. Hence $\phi_{1}^{a}=G$ for every shape in the chain. Therefore, for any $\left(n, g, s^{\prime}, k^{\prime}\right)$-shape group, $\phi_{1}^{a}=G$.

Now I state a series of remarks which shows which shapes can be expanded into which.

Remark 2. Let $s<n-1$. $(n, n, s, 0)$ is expandable to $(n, n, s+1, s+1)$.

Proof. Consider any $(n, n, s+1, s+1)$-shape group $(I, G, V)$, and consider the set $V_{s+1}^{b}-V_{s+2}^{b}$. By definition of the shape, there are 2 goods in this set, called, say, $g_{1}$ and $g_{2}$. Removing either $g_{1}$ or $g_{2}$ from $V_{s+1}^{b}$ gives a $(n, n, s, 0)$-shape.

Remark 3. $(n, n, n-1,0)$ is expandable to $(n, n, n, 0)$.

PROOF. Removing any one of the $n$ goods in $V_{n}^{b}$ from a $(n, n, n, 0)$-shape group gives a $(n, n, n-1,0)$-shape group.

Remark 4. Let $s \leq k<n-1$. $(n, n, s, k)$ is expandable to $(n, n, s, k+1)$.

Proof. Consider a $(n, n, s, k+1)$-shape group and the set $V_{k+1}^{b}-V_{k+2}^{b}$. By definition, there are two goods in this set. Removing either good from $V_{k+1}^{b}$ gives a $(n, n, s, k)$-shape.

Remark 5. Let $s \geq 2$. $(n, n, s, n-1)$ is expandable to $(n, n, s, 0)$.

Proof. Consider a $(n, n, s, 0)$-shape group and the set $V_{n}^{b}$. By the shape definition and the condition $s \geq 2$, there are at least 2 goods in $V_{n}^{b}$. Removing either good from $V_{n}^{b}$ gives a $(n, n, s, n-1)$-shape.

I am now ready to show that for any rectangular isolated group of size $n$ the highest-budget bidder wins all the goods.

Remark 6. For any $(n, n, n, 0)$-shape group $(I, G, V), \phi_{1}^{a}=G$.

Proof. By remark 2, $(n, n, 1,0)$ is expandable to $(n, n, 2,2)$. By remark $4,(n, n, 2,2)$ is expandable to $(n, n, 2,3)$ which is in turn expandable to $(n, n, 2,4)$ and so on until $(n, n, 2, n-1)$. By remark $5,(n, n, 2, n-1)$ is expandable to $(n, n, 2,0)$. In other words, $(n, n, 1,0)$ is chain-expandable to $(n, n, 2,0)$.

This line of reasoning can be repeated to show that $(n, n, 1,0)$ is chain-expandable to $(n, n, n-1,0)$. By remark 3 , $(n, n, n-1,0)$ is expandable to $(n, n, n, 0)$. Therefore $(n, n, 1,0)$ is chain-expandable to $(n, n, n, 0)$.

By remark 1 and the inductive hypothesis that for any $(n, n, 1,0)$-shape group $(I, G, V), \phi_{1}^{a}=G$, it must be true that for any $(n, n, n, 0)$-shape group $(I, G, V) \phi_{1}^{a}=G$. 
Remark 6 shows that a triangle shape can be expanded to a square shape while preserving the allocation rule that all goods are given to the bidder with the highest budget. I now show that a triangle shape can also be expanded to a larger triangle.

Remark 7. For any $(n+1, n+1,1,0)$-shape group $(I, G, V), \phi_{1}^{a}=G$.

Proof. Because $(n, n, 1,0)$ is expandable to $(n, n, 2,0)$, for any $(n, n, 2,0)$-shape group $\left(I^{1}, G^{1}, V^{1}\right), \phi_{1}^{a}=G^{1}$.

Consider a $(n+1, n, 2,0)$-shape group $\left(I^{2}, G^{2}, V^{2}\right)$. Suppose bidder $n+1$ is winning some bundle $x_{n+1} \in V_{n+1}^{2}$. By construction, $V_{n+1}^{2} \subseteq V_{n}^{2} \subseteq \ldots \subseteq V_{1}^{2}$, so by lemma 7 , all $n+1$ bidders must be winning. There are however only $n$ goods, so this cannot hold.

Therefore bidder $n+1$ is losing. If this bidder reports valuation of zero for all bundles, then non-wastefulness he is still losing and by non-bossiness he does not change the allocation of other bidders. In particular, allocation for bidder 1 must not be changed. However, with such a report the remaining bidders and the goods constitute a $(n, n, 2,0)$-shape group for which bidder 1 wins all the goods. Therefore for the original $(n+1, n, 2,0)$-shape group $\left(I^{2}, G^{2}, V^{2}\right)$, bidder 1 must still win all the goods, i.e., $\phi_{1}^{a}=G^{2}$.

Now consider a group $(I, G, V)$ of $(n+1, n+1,1,0)$-shape. Let $g_{n+1}=V_{1}^{b}-V_{2}^{b}$ be the good valued only by bidder 1. If bidder 1 reports $\hat{V}_{1}=V_{2}$ then the bidders and the goods constitute a $(n+1, n, 2,0)$-shape group $\left(I, G-g_{n+1}, \hat{V}\right)$, at which, by the above, bidder 1 wins $G-g_{n+1}$. The good $g_{n+1}$ is now worthless to all bidders, so when bidder 1 reports $V_{1}$, by lemma 5, bidder 1 must win $G-g_{n+1}+g_{n+1}$. So bidder 1 wins all the goods in $G$.

I have shown that, given the inductive hypothesis that the highest-budget bidder wins all goods for any $(n, n, 1,0)$ shape group, the same allocation rule holds for any $(n+1, n+1,1,0)$-shape group and any $(n, n, n, 0)$-shape group. By induction and the fact that the allocation rule holds for the base case of shape $(2,2,1,0)$, the allocation rule holds true for any $(n, n, 1,0)$-shape and any $(n, n, n, 0)$-shape for any $n$. This is summarized by the following remark.

Remark 8. Let $n$ be any natural number. For any $(n, n, 1,0)$-shape group $(I, G, V), \phi_{1}^{a}=G$. For any $(n, n, n, 0)$-shape group $(I, G, V), \phi_{1}^{a}=G$.

It it straightforward to extend the allocation rule to rectangular shape groups by adding or removing losing bidders.

Remark 9. For any $n, g$, for any $(n, g, n, 0)$-shape group $(I, G, V), \phi_{1}^{a}=G$.

PROOF. There are 3 cases: $n=g, n>g$ and $n<g$. For the case $n=g$, one can apply claim 8 .

Consider the case $n>g$. Consider bidder $k$ where $g+1 \leq k \leq n$. If bidder $k$ is winning, then by the structure of value sets and lemma 7, for any $i<k$, bidder $i$ is winning. This means there are at least $g+1$ winning bidders but there are only $g$ goods, an impossibility. Hence bidder $k$ must be losing.

Consider bidder $n$. By the above, bidder $n$ is losing, so changing bidder $n$ 's valuation for all bundles to zero keeps bidder $n$ losing and by non-bossiness does not change the allocation of other bidders. Repeat the above for bidder $n-1$, and then bidder $n-2$ and so on until all bidders labeled from $g+1$ to $n$ have zero valuation for all bundles. The remaining bidders and the goods now constitute a $(g, g, g, 0)$-shape group for which, by remark 8 , bidder 1 wins all the goods. Non-bossiness implies that before the changes, $\phi_{1}^{a}=G$.

For the case where $n<g$, first consider a $(g, g, g, 0)$-shape group. For this group, by claim $8, \phi_{1}^{a}=G$, so for all $i \neq 1, \phi_{i}^{a}=\emptyset$. For $i \geq n$, changing bidder $i$ 's valuation for all bundles to zero keeps bidder $i$ losing and by non-bossiness preserves the allocation $\phi_{1}^{a}=G$. Doing this iteratively for all such bidders lead to a $(n, g, n, 0)$-shape group. Since any $(n, g, n, 0)$-shape group can be arrived at in this manner, it must be that for any $(n, g, n, 0)$-shape group, $\phi_{1}^{a}=G$.

Noting that any isolated rectangular group must take the shape of $(n, g, n, 0)$ for some $n$ and $g$ establishes the result that for any isolated rectangular group, the highest bidder wins all the goods in the group. 